Draft VERSion July 2, 2019

Typeset using LATEX twocolumn style in AASTeX63

\title{
APOGEE/Kepler Overlap Yields Orbital Solutions for a Variety of Eclipsing Binaries
}

\author{
Joni Marie Clark Cunningham, ${ }^{1,2}$ Meredith L. Rawls, ${ }^{3,4}$ Diana Windemuth, ${ }^{3}$ Aleezah Ali,${ }^{3}$ Jason Jackiewicz, ${ }^{5}$ \\ Eric Agol, ${ }^{3}$ Keivan G. Stassun, ${ }^{2,1}$ \\ ${ }^{1}$ Department of Physics, Fisk University, Nashville, TN 37208 \\ ${ }^{2}$ Department of Physics and Astronomy, Vanderbilt University, Nashville, TN 37235 \\ ${ }^{3}$ Department of Astronomy, University of Washington, Seattle, WA 98195 \\ ${ }^{4}$ DIRAC Institute, University of Washington, Seattle, WA 98195 \\ ${ }^{5}$ Department of Astronomy, New Mexico State University, Las Cruces, NM 88003
}

\begin{abstract}
Spectroscopic Eclipsing Binaries (SEBs) are fundamental benchmarks in stellar astrophysics and today are observed in breathtaking detail by missions like TESS, Kepler, and APOGEE. We develop a methodology for simultaneous analysis of high precision Kepler light curves and high resolution near-IR spectra from APOGEE and present orbital solutions and evolutionary histories for a subset of SEBs within this overlap. Radial velocities extracted from APOGEE spectra using the Broadening Function technique are combined with Kepler light curves and to yield binary orbital solutions. The Broadening Function approach yields more precise radial velocities than the standard Cross-Correlation Function, which in turn yields more precise orbital parameters and enables the identification of tertiary stars. The orbital periods of these seven SEBs range from 4 to 40 days. Four of the systems (KIC 5285607, KIC 6864859, KIC 6778289, and KIC 4285087) are well-detached binaries. The remaining three systems have apparent tertiary companions, but each exhibits two eclipses along with at least one spectroscopically varying component (KIC 6449358, KIC 6131659, and KIC 6781535). Gaia distances are available for four targets which we use to estimate temperatures of both members of these SEBs. We explore evolutionary histories in H-R diagram space and estimate ages for this subset of our sample. Finally, we consider the implications for the formation pathways of close binary systems via interactions with tertiary companions. Our methodology combined with the era of big data and observation overlap opens up the possibility of discovering and analyzing large numbers of diverse SEBs, including those with high flux ratios and those in triple systems.
\end{abstract}

\section{INTRODUCTION}

The Apache Point Observatory Galactic Evolution Experiment (APOGEE) is studying our Galaxy in fantastic detail by providing high resolution spectra for some 150,000 stars (Majewski et al. 2015). Some of these belong to double-lined spectroscopic eclipsing binaries (SEBs), and a further subset have been observed by the Kepler spacecraft (Borucki et al. 2010) and appear in the Kepler Eclipsing Binary Catalog (Kirk et al. 2016). These APOGEE/Kepler SEBs which have several APOGEE spectra at different epochs give a unique opportunity to combine the spectra with the Kepler light curve to model the binary orbit and directly measure fundamental stellar parameters, including mass and radius. They can then be used to explore and constrain stellar evolution, stellar populations, and orbital kinematics.

While much work has gone into exploring Kepler eclipsing binaries (EBs) as a population, fewer studies have maximally utilized complementary spectra to fully characterize these stellar systems. A notable exception is Matson et al. (2017), which found the radial velocities of 40 Kepler binaries, 35 of them doublelined and the remainder single-lined. Their work used medium resolution ground-based spectra, but the authors note that high resolution spectra is more optimal. In another example, Torres et al. (2018) used K2 light curves of the Ruprecht 147 cluster together with high resolution spectra and the cluster's well-modeled metallicity to constrain the orbital parameters extracted from spectroscopic binary cluster members. In addition, Lehmann et al. (2012) analyzed the quadruple system KIC 4247791 by combining Kepler light curves and moderate resolution spectra.

Many studies have used APOGEE and Kepler data together, such as the APOKASC catalog (Pinsonneault et al. 2018, 2014) which combines APOGEE stellar parameters with Kepler asteroseismology. However, such works tend to ignore stellar multiplicity. The SEB overlap between APOGEE and Kepler in particular remains 
relatively unexplored. There is also synergy with the Kepler planet survey which identifies candidate planet systems, some of which are found to be eclipsing binaries, with or without tertiaries, with follow-up radialvelocity observations with APOGEE (Fleming et al. 2015). The frequency of binaries, with and without tertiary companions, is a necessary component of computing transiting exoplanet astrophysical false-alarm probabilities (e.g. Morton et al. 2016).

EBs have long been used as fundamental benchmarks for stellar astrophysics (e.g., Torres et al. 2010), including more recently as benchmarks for exoplanet properties (e.g., Stassun et al. 2017), to test asteroseismic inferences of stellar parameters (e.g., Gaulme et al. 2016), and even for assessing trigonometric parallaxes (Stassun \& Torres 2018). In addition, as they are often observed as SEBs, EBs are useful for assembling reliable statistics on the occurrence of higher order multiples (e.g., tertiary companions) and on the relationship of companion properties to the properties of the EB. For example, Tokovinin (1997) found the incidence of wide tertiaries to be strongly linked to the orbital period of the inner binary. Additional well-studied EBs can help to further test these relationships.

In this work, we identify 33 promising APOGEE/Kepler SEBs and compute full orbital solutions with a suite of stellar parameters for seven of them. In $\$ 2$, we detail our sample selection, data processing, and modeling methodology. We further show how the Broadening Function technique is a superior method to extract multiple velocity components from APOGEE spectra. Subsequently $\$ 3$ discusses each of the seven modeled systems in turn and presents orbital solutions. Finally, \$4 places the SEBs in the context of each star's stellar evolutionary history and explores the relationship of the EB orbital properties to the presence of tertiary companions.

\section{DATA AND METHODS}

\subsection{Sample Selection}

We use the following criteria and filters to arrive at a candidate sample of promising SEBs in the APOGEE/Kepler overlap. We begin with the Kepler EB catalog compiled by Kirk et al. (2016). From this catalog we select targets which have both their primary and secondary eclipses observed by Kepler; this limits our selection to binaries with inclinations close to 90 degrees. We further require the light curve to be semi- or well-detached, with the morphology parameter significantly less than 1 . Next, a luminosity limit of $H<14$ magnitudes was imposed, as fainter targets are unlikely to have $H$-band APOGEE spectra with a sufficiently high signal-to-noise ratio. We also require the targets to have multiple cross-correlation function (CCF) peaks from the APOGEE pipeline (Nidever et al. 2015) visible by eye in one epoch. Finally, the binaries must have been observed by APOGEE at least three times, and thus have at least three apVisit spectra, with no quality flags present.

Taken together, these criteria result in 33 candidates, which are listed in Table 2, plus one additional candidate which has already been analyzed (Rawls et al. 2016). Of these, we perform a detailed analysis of seven. Notes in Table 2 indicate why we choose to exclude the other systems at this time. Several are being investigated by the Kepler APOGEE EB Working Group, some have only three APOGEE visits which would make a good RV curve solution challenging without additional spectra, some have low signal-to-noise $(\mathrm{S} / \mathrm{N})$ ratios, one shows significant ellipsoidal variations which are not included in our photometric model, and two remain good candidates for future analyses.

\subsection{Radial Velocities (RVs) from APOGEE Spectra}

The standard observing mode for APOGEE spectra has a total exposure time of roughly three hours, which is usually collected over a series of visits on different days. The visits are then combined into one spectrum per target (an apStar spectrum). We instead utilize individual visit spectra (apVisit), which are identified with their plate ID, date (MJD), and fiber ID. These may be retrieved from the SDSS Science Archive Server search tool with a simple search by APOGEE ID. We continuum normalize the visit spectra and then "despike" them to remove erroneous spectral features due to tellurics. De-spiking consists of identifying outliers above or below the continuum by 0.7 or 3 times the standard deviation of the normalized flux, respectively. The "below continuum" factor is larger to avoid unintentionally removing real absorption line features. Around each outlier spike, a $\pm 6 \AA$ window is also flagged for removal. The python scripts used to retrieve, continuum normalize, and de-spike apVisit spectra are publicly available on GitHul[. They rely heavily on the apogee python package on GitHub described in Bovy (2016).

In the main APOGEE reduction pipeline (Nidever et al. 2015), RVs are measured using the CCF. In this approach, a template spectrum and a series of visit spectra for a given target are cross-correlated, giving the RV of the target star relative to the template.

The CCF method works because an observed stellar spectrum can be represented as a convolution of two

\footnotetext{
${ }^{1}$ https://github.com/mrawls/apVisitproc
} 
Table 2. Promising SEBs observed by APOGEE and Kepler, sorted by Kepler magnitude $\left(K_{p}\right)$

\begin{tabular}{|c|c|c|c|c|c|c|c|c|}
\hline $\mathrm{KIC}$ & APOGEE ID & Visits & $K_{p}$ & $P_{\text {orb }}$ (day) & SE Depth (frac) & Morphology & Reference & Notes \\
\hline 9246715 & $2 \mathrm{M} 20034832+4536148$ & 2 & 10.08 & 171.28 & 0.1124 & 0.11 & Rawls et al. $(\overline{2016})$ & \\
\hline 2708156 & $2 \mathrm{M} 19302686+4318185$ & 3 & 10.67 & 1.89 & 0.0625 & 0.57 & & Only 3 visits \\
\hline 3120320 & $2 \mathrm{M} 19291007+3817041$ & 3 & 11.28 & 10.27 & 0.0127 & 0.14 & & Kepler APOGEE/EB WG \\
\hline 4851217 & $2 \mathrm{M} 19432016+3957081$ & 6 & 11.32 & 2.47 & 0.1815 & 0.58 & & Low $\mathrm{S} / \mathrm{N}$ ratio \\
\hline 3439031 & $2 \mathrm{M} 19203184+3830492$ & 3 & 11.50 & 5.95 & 0.4156 & 0.33 & & Kepler APOGEE/EB WG \\
\hline 5285607 & $2 \mathrm{M} 19390532+4027346$ & 6 & 11.69 & 3.90 & 0.0403 & 0.36 & This work & \\
\hline 6449358 & $2 \mathrm{M} 19353513+4149543$ & 25 & 11.72 & 5.78 & 0.0120 & 0.31 & This work & \\
\hline 10206340 & $2 \mathrm{M} 19245882+4714573$ & 3 & 11.78 & 4.56 & 0.2431 & 0.61 & & Only 3 visits \\
\hline 6864859 & $2 \mathrm{M} 19292405+4223363$ & 25 & 11.93 & 40.88 & 0.2426 & 0.06 & This work & \\
\hline 4931073 & $2 \mathrm{M} 19351913+4001522$ & 6 & 12.18 & 26.95 & 0.0564 & 0.08 & & Kepler APOGEE/EB WG \\
\hline 3127817 & $2 \mathrm{M} 19355993+3813561$ & 6 & 12.24 & 4.33 & 0.0512 & 0.48 & & Kepler APOGEE/EB WG \\
\hline 3335816 & $2 \mathrm{M} 19184759+3824238$ & 3 & 12.40 & 7.42 & 0.0106 & 0.16 & & Kepler APOGEE/EB WG \\
\hline 5284133 & $2 \mathrm{M} 19373173+4027078$ & 6 & 12.50 & 8.78 & 0.0492 & 0.15 & & Future work \\
\hline 3542573 & $2 \mathrm{M} 19232622+3838017$ & 3 & 12.61 & 6.94 & 0.0837 & 0.25 & & Only 3 visits \\
\hline 2711114 & $2 \mathrm{M} 19240341+3758109$ & 3 & 12.63 & 2.86 & 0.0022 & 0.29 & & Only 3 visits \\
\hline 4281895 & $2 \mathrm{M} 19441242+3923418$ & 6 & 12.76 & 9.54 & 0.0652 & 0.13 & & Only 3 visits \\
\hline 4660997 & $2 \mathrm{M} 19340328+3942410$ & 6 & 12.78 & 0.56 & 0.2527 & 0.62 & & Ellipsoidal variations \\
\hline 4473933 & $2 \mathrm{M} 19363898+3933105$ & 6 & 12.87 & 103.59 & 0.0126 & 0.25 & & Low $\mathrm{S} / \mathrm{N}$ ratio \\
\hline 2305543 & $2 \mathrm{M} 19280644+3736023$ & 3 & 12.97 & 1.36 & 0.1052 & 0.50 & & Only 3 visits \\
\hline 3241619 & $2 \mathrm{M} 19322278+3821405$ & 3 & 13.06 & 1.70 & 0.1625 & 0.44 & & Only 3 visits \\
\hline 4285087 & $2 \mathrm{M} 19463571+3919069$ & 6 & 13.19 & 4.49 & 0.2408 & 0.31 & This work & \\
\hline 2576692 & $2 \mathrm{M} 19263432+3748513$ & 3 & 13.19 & 87.88 & 0.2588 & 0.04 & & Kepler APOGEE/EB WG \\
\hline 6131659 & $2 \mathrm{M} 19370697+4126128$ & 27 & 13.20 & 17.53 & 0.1036 & 0.09 & This work & \\
\hline 4847832 & $2 \mathrm{M} 19401839+3957298$ & 6 & 13.20 & 30.96 & 0.3200 & 0.08 & & Kepler APOGEE/EB WG \\
\hline 5025294 & $2 \mathrm{M} 19414825+4010323$ & 6 & 13.27 & 5.46 & 0.0010 & 0.18 & & Future work \\
\hline 6778289 & $2 \mathrm{M} 19282456+4215080$ & 25 & 13.31 & 30.13 & 0.1619 & 0.11 & This work & \\
\hline 3248332 & $2 \mathrm{M} 19383951+3819588$ & 6 & 13.37 & 7.36 & 0.0974 & 0.20 & & Kepler APOGEE/EB WG \\
\hline 6610219 & $2 \mathrm{M} 19320615+4200049$ & 25 & 13.58 & 11.30 & 0.2899 & 0.20 & & Low $\mathrm{S} / \mathrm{N}$ ratio \\
\hline 6781535 & $2 \mathrm{M} 19321788+4216489$ & 25 & 14.14 & 9.12 & 0.0305 & 0.12 & This work & \\
\hline 4077442 & $2 \mathrm{M} 19452193+3908260$ & 6 & 14.35 & 0.69 & 0.0703 & 0.59 & & Kepler APOGEE/EB WG \\
\hline 3247294 & $2 \mathrm{M} 19374558+3822510$ & 6 & 14.35 & 67.42 & 0.1032 & 0.02 & & Kepler APOGEE/EB WG \\
\hline 3848919 & $2 \mathrm{M} 19241352+3858278$ & 3 & 14.48 & 1.05 & 0.3418 & 0.57 & & Low $\mathrm{S} / \mathrm{N}$ ratio \\
\hline 5460835 & $2 \mathrm{M} 19411125+4039416$ & 6 & 14.72 & 21.54 & 0.0228 & 0.06 & & Low $\mathrm{S} / \mathrm{N}$ ratio \\
\hline 4075064 & $2 \mathrm{M} 19432862+3908535$ & 6 & 15.71 & 61.42 & 0.0821 & 0.00 & & Low $\mathrm{S} / \mathrm{N}$ ratio \\
\hline
\end{tabular}

functions: that of the astrophysical target (which includes "natural broadening" components such as thermal broadening, microturbulence effects, and instrumental broadening) and another function called the Broadening Function (BF) that contains the important $\mathrm{RV}$ information. The BF is formally presented in Rucinski (1992, 1999, 2002, 2004). Although the CCF method is very close to the real convolution that occurs in an APOGEE spectrum, cross-correlating a template spectrum with an observed stellar spectrum yields a function which inherits the natural broadening components present in both spectra. In this way the CCF is essentially a non-linear proxy of the BF. Therefore, instead of using the $\mathrm{CCF}$, in this work we measure the BF directly.

To extract BFs from our target spectra, we use a modified version of the BF software suite from Rawls et al. (2016) which is based on the method introduced by Rucinski (1992). A PHOENIX BT-Settl model atmosphere spectrum (Husser et al. 2013) is selected to match the target's approximate spectral parameters as reported by APOGEE. The match cannot be exact as the two stars in a binary may not have identical spectral types and the model grid has a finite sampling in stellar parameters. In general, a mismatch in spectral type between template and target causes the $\mathrm{BF}$ to change in its intensity scale and quality, but the amplitudes of the RV components remain unchanged (Lu et al. 2001).

We examine the BF peaks by eye to identify their approximate locations on the radial velocity axis and use a least-squares fitting procedure to fit one or more Gaussians to the BF. The location of each Gaussian's mean is the RV, which we then correct with the barycentric velocity provided with each apVisit spectrum. Our reported RV uncertainties come from the error in fitting a Gaussian to each BF peak using least-squares. Much like in the APOGEE CCF pipeline (Nidever et al. 2015), it is ultimately the uncertainty in the measurement of the BF peak, which depends partially on its semi-arbitrary 
width, that determines the reported uncertainties for the RVs. This systematically underestimates the uncertainty of each RV measurement. The software used to extract RVs as described here is publicly available on GitHub.

In Figures 1 and 2, we demonstrate how the BF method produces significantly better separated peaks for APOGEE double-lined SEBs than the CCFs generated by the APOGEE pipeline. Due in part to the BF method having less of a "peak-pulling" effect, this more defined separation dramatically improves our ability to measure the RV of each component. We present the $\mathrm{BF}$ and measured RVs for each of our seven targets in Appendix A.

We also measure flux ratios in the APOGEE $H$ band with our BF peaks, as the ratio of the peak areas is directly proportional to the flux ratio of the binary (Bayless \& Orosz 2006; Stassun et al. 2007). These BF flux ratios will generally differ from Kepler-derived flux ratios because of the difference in wavelength; APOGEE is an $H$-band spectrograph and Kepler has a broad visible light bandpass. The BF flux ratios are discussed further in Section 3.8 alongside other variables for RV extraction and temperature estimation.

\subsection{Kepler Light Curve Processing}

We use minimally-processed Kepler long-cadence simple aperture photometry (SAP) to construct EB light curves for each target. Each light curve, and its uncertainties, is normalized by the median raw flux value of all available quarters. We de-weight data of poor quality by selecting times with Kepler quality flags $>0$, and inflating the normalized flux uncertainties at these times by a factor of 10 .

\subsection{Orbital and Mass Solutions with KEBLAT}

With RVs in hand, we turn to the photometric modeling of the Kepler light curves. We utilize a modular Python tool dubbed "KEBLAT" which is capable of separate or simultaneous modeling of the binary light curve, spectral energy distribution (SED), and RV time series (Windemuth et al. 2018). Here, we simultaneously model Kepler light curves and APOGEE RVs of each EB in our sample to determine orbital solutions $\left(P, e, \omega, i, t_{P E}\right)$, stellar parameters $\left(m_{1}, m_{2}, r_{1}, r_{2}, \frac{F_{2}}{F_{1}}\right)$, quadratic limb darkening coefficients under triangular reparameterization $\left(q_{1,1}, q_{1,2}, q_{2,1}, q_{2,2}\right.$; Kipping 2013), and systemic radial velocity $k_{0}$. For parameter sampling purposes, we transform individual mass and radius parameters to sums and ratios, and parameterize $e$ and $\omega$ as $e \cos \omega$ and $e \sin \omega$.

Given a system's total mass, period, eccentricity, argument of periastron, inclination, and time of primary eclipse, KEBLAT uses a Keplerian solver to compute the instantaneous positions and velocities of each stellar component. The positions, along with specified sizes and relative flux of the stars are then used to determine the instantaneous light contribution during eclipse via a quadratic limb-darkening (Mandel \& Agol 2002) model for spherical bodies ${ }^{2}$ We account for finite sampling effects (Kipping 2010) on the light curve by downsampling 1-minute eclipse profiles to the Kepler long cadence $(d t=0.0204 \mathrm{~d})$. Stellar and instrumental noise is marginalized by fitting the lowest non-linear order quadratic polynomial around each eclipse. We apply quarterly crowding values from Kepler to model third light contamination. To account for underestimated observational uncertainties and additional noise, we fit for a systematic light curve error $\sigma_{\mathrm{sys}, \mathrm{LC}}$, which we add in quadrature to the observed errors.

The z-component of the velocity, as solved by Kepler's equation, is used to model the extracted RVs. For double-line eclipsing binary systems, where the RVs of both components are detected, the amplitudes of the primary and secondary RV are related to the masses of the secondary and primary, respectively. For single-line $\mathrm{EBs}$, where only the RV of the brightest component is detected, only the "mass function" $f_{M}$ of the system can be constrained, where

$$
f_{M}=\frac{M_{2}^{3} \sin ^{3} i}{\left(M_{1}+M_{2}\right)^{2}}
$$

As with the light curve data, we fit for a systematic radial velocity error parameter $\sigma_{\text {sys,Rv }}$ to account for underestimated noise.

We combine RVs with Kepler light curve information to model the system and find a best fit solution. We first determine the light curve and RV solutions separately, and then fit RV and light curve simultaneously. The simultaneous RV+LC model has 17 free parameters in total. The model is optimized via a least-squares algorithm lmfit (Newville et al. 2016), and then uses the best-fit solution to seed Monte Carlo Markov Chain (MCMC) simulations with emcee (Foreman-Mackey et al. 2013), in order to sample the posterior distributions of each parameter. We use broad, uniform priors and run the Markov chains with 128 walkers for $\sim 100,000$ iterations, visually inspecting trace plots for convergence. We report the $50 \%, 16 \%$, and $86 \%$ values, i.e., the mean and $1 \sigma$ uncertainties for each parameter. For more details on the KEBLAT model, including parameter bounds, see Windemuth et al. (2018).

\footnotetext{
2 The assumption of spherical stars requires that the stars be sufficiently detached to avoid tidal and rotational distortions.
} 


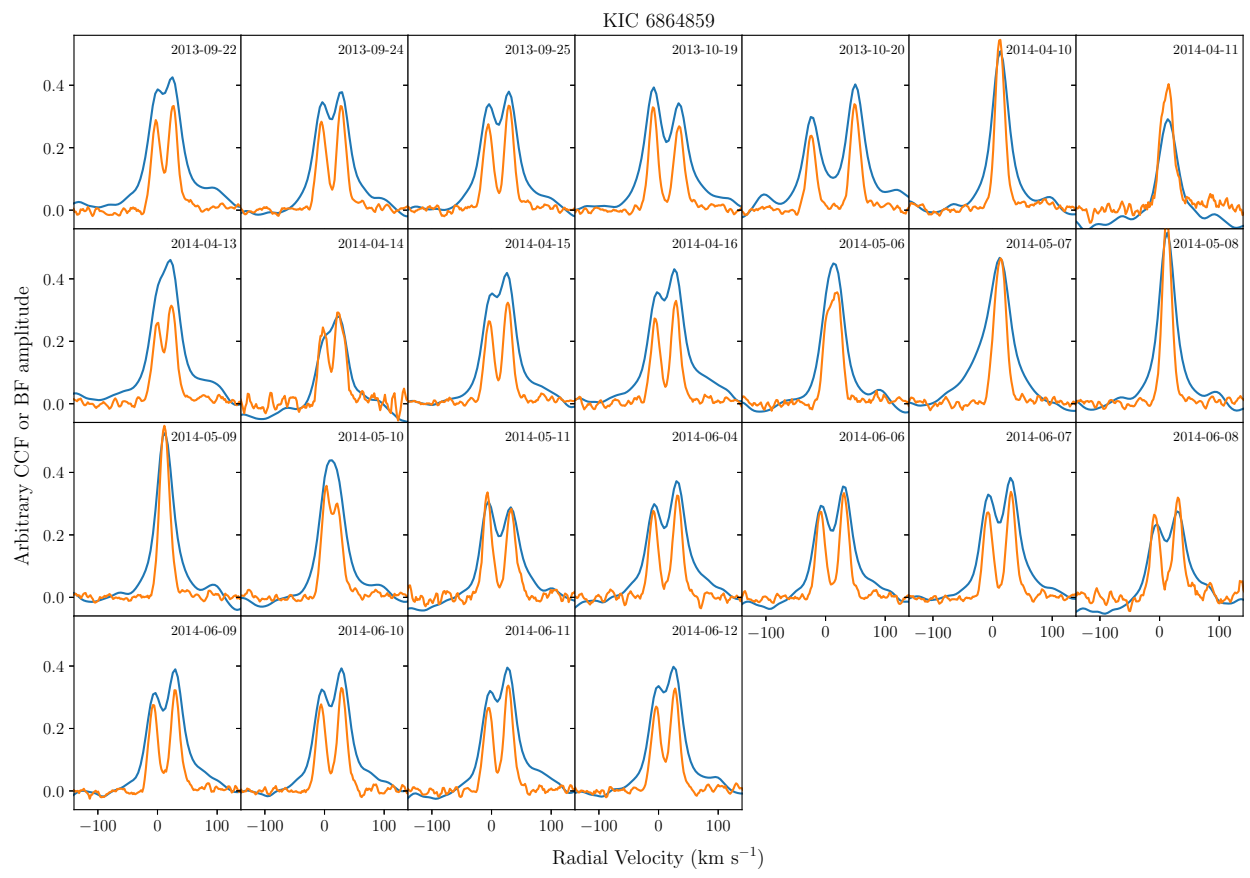

Figure 1. The BF (orange) is significantly better at resolving multiple velocity components from APOGEE visit spectra than the CCF (blue). This example shows visits for KIC 6864859. The y-axis amplitude is arbitrarily scaled for clarity. While it is clear from most of the CCFs that KIC 6864859 is a double-lined SEB, the BF more clearly separates the contribution from each star.

\subsection{Radius ratio-flux ratio-inclination Degeneracy}

For light curves with partially or grazing eclipsing geometries, there exists a degeneracy between radius ratio and flux ratio when eclipses are observed in a single photometric band. For this reason, we use additional constraints on the Kepler light curve flux ratios with spectroscopic $H$-band flux ratios obtained from the $\mathrm{BF}$ for SEBs exhibiting shallow eclipses (KIC 5285607 and KIC 6781535). For these two systems, we place a Gaussian prior on the RV $+\mathrm{LC}$ solution with $\mu$ centered around the BF-derived flux ratio and $\sigma=0.2$.

\subsection{Temperatures from Flux Ratios and Radii}

Obtaining effective temperatures of the stars in these SEBs requires additional analysis. The KEBLAT model does not provide a measure of stellar temperatures directly, but only indirectly via the flux ratio in the $\mathrm{Ke}$ pler bandpass. In addition, the APOGEE Stellar Parameter and Chemical Abundances Pipeline (ASPCAP) processing reports only a single "combined light" effective temperature for each system (Pérez et al. 2016). This ASPCAP temperature is likely to be biased due to the contamination of the brighter star's spectrum by the fainter star. Here we address both of these issues to estimate individual stellar effective temperatures.

The orbital solutions described in $\$ 2.4$ yield sums and ratios of radii. In addition, the light curve analyses yield flux ratios $\left(F_{\text {ratio }} \equiv \frac{F_{2}}{F_{1}}\right)$ in the Kepler bandpass which are primarily constrained by the observed eclipse depths.

By assuming the ASPCAP effective temperature is the flux weighted average of the system, $T_{\text {avg }}=\left(F_{1} T_{1}+\right.$ $\left.F_{2} T_{2}\right) /\left(F_{1}+F_{2}\right)$, and defining the primary star as the one that provides the majority of the light, we can use the following relationships between the flux ratio $F_{\text {ratio, }}$, $T_{\mathrm{avg}}$, stellar radii $R_{1}$ and $R_{2}$, and distance $d$ to find flux and temperature estimates for the individual binary components separately. First, we solve for the binary's flux sum using Gaia distance estimates from Bailer-Jones et al. (2018):

$$
F_{\text {sum }}=\frac{\sigma T_{\mathrm{avg}}^{4}\left(R_{1}^{2}+R_{2}^{2}\right)}{d^{2}}
$$

from which we can compute the individual fluxes as

$$
F_{1}=\frac{F_{\text {sum }}}{1+F_{\text {ratio }}}
$$

and

$$
F_{2}=F_{\text {sum }}-F_{1} .
$$




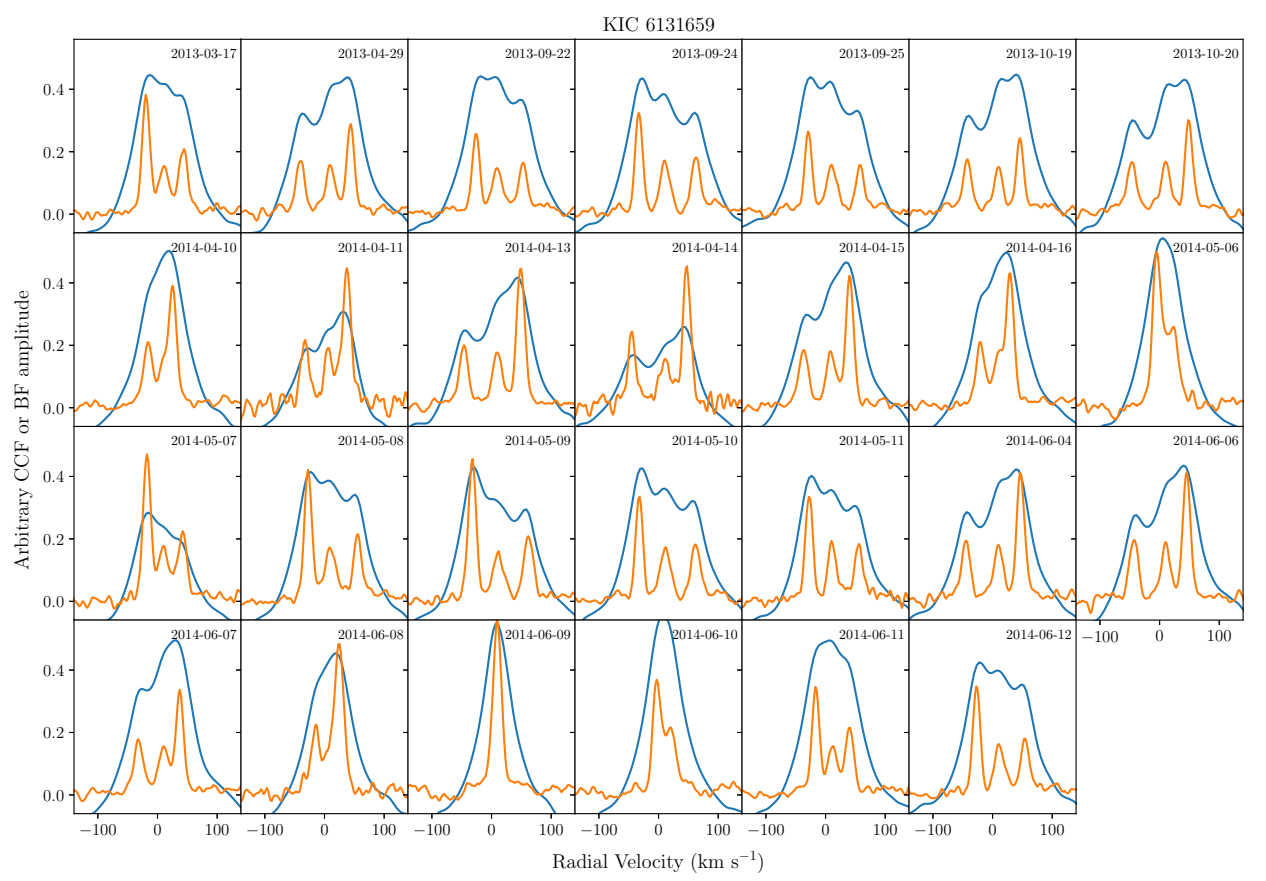

Figure 2. A second example, as in Figure 1, showing that the BF (orange) does a better job of resolving multiple velocity components than the CCF (blue). The y-axis amplitude is arbitrarily scaled for clarity. While it is clear from some of the CCFs that KIC 6131659 has more than one component, the three-component nature is immediately obvious in the BF. In all but a few cases, it is straightforward to precisely measure the RV of all three BF peaks. This is not true for any visit using the APOGEE CCFs.

Then, we use the relationship between $T_{\text {ave }}, F_{\text {ratio }}$, and the individual stellar fluxes to solve for the temperature of each star:

$$
T_{\text {avg }}=T_{2} \frac{F_{\text {ratio }}^{-1} T_{\text {ratio }}^{-1}+1}{F_{\text {ratio }}^{-1}+1},
$$

which yields

$$
T_{1}=\sqrt[4]{\frac{F_{1} d^{2}}{\sigma R_{1}^{2}}}
$$

and

$$
T_{2}=\sqrt[4]{\frac{F_{2} d^{2}}{\sigma R_{2}^{2}}}
$$

However, these resulting temperature estimates are likely systematically underestimated. In the ASPCAP pipeline, APOGEE spectra are compared to a synthetic spectral model to resolve quantities like effective temperature. When a detached binary signature is present in stellar spectra, the additional component can cause the spectrum to be fit by a cooler synthetic template. This leads to a systematic underestimation in the binaries' effective temperatures of roughly $300 \mathrm{~K}$ (El-Badry et al. 2017). The systematic underestimation is a function of the effective temperature of the primary and the mass ratio of the system. We follow their method for each system to correct for this effect.

\section{RESULTS}

The joint light curve and RV analysis for each of the seven systems is presented in detail in the following subsections. We present orbital and mass solutions together in Table 3 ,

In all systems, we define the primary eclipse $(\phi=0)$ as the deeper eclipse. This corresponds to when the primary star is eclipsed by the secondary star. Usually, the primary star is the brighter of the two, and the light curve definition of primary and the RV definition of primary agree. However, we note the secondary star in KIC 6781535 is brighter in the APOGEE $H$-band than the primary. In the figures that follow, we color code the RV of the primary star in red and the RV of the secondary star in orange. 


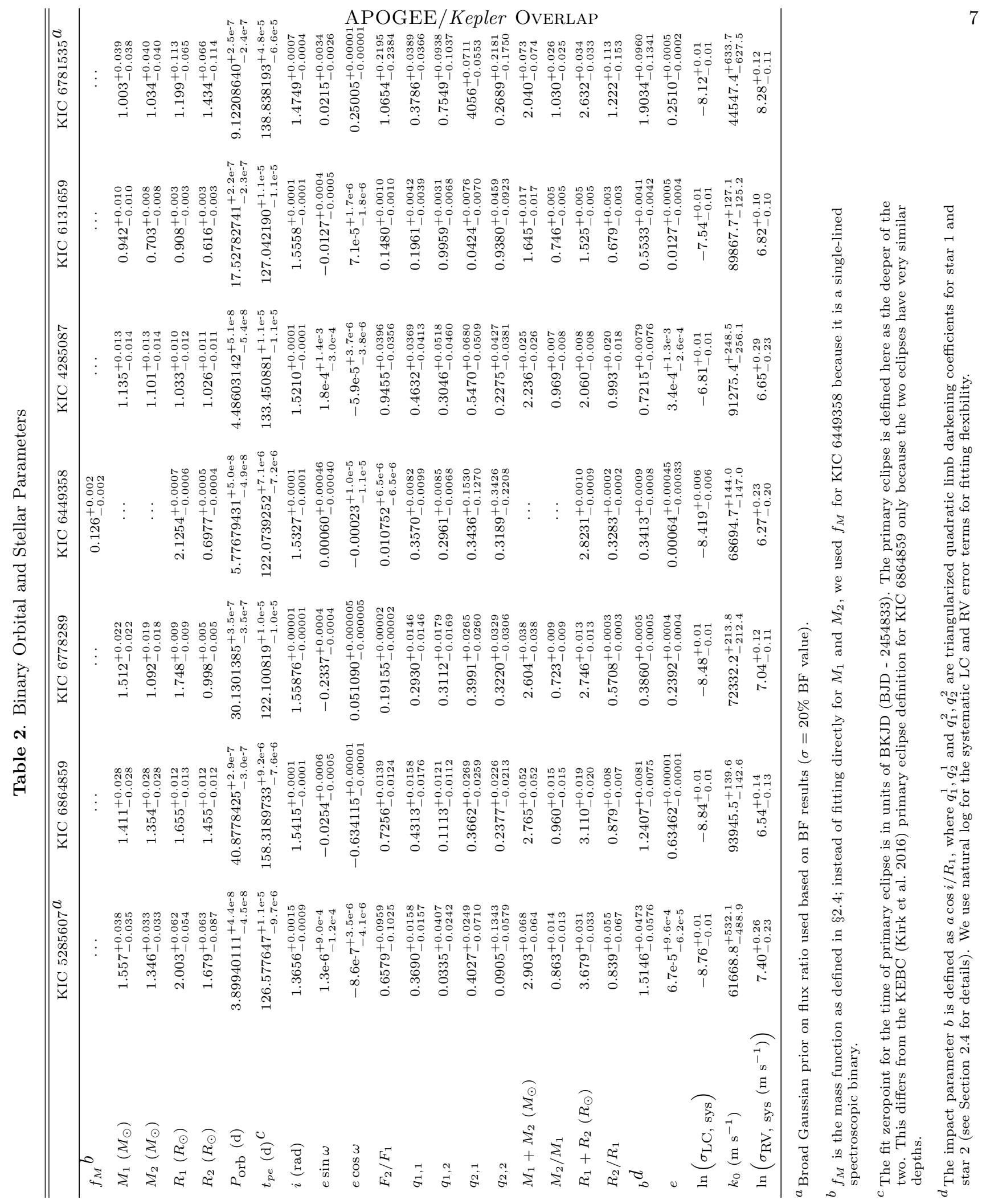




\subsection{KIC 5285607}

KIC 5285607 is a grazing $\left(i=79^{\circ}\right) 3.9 \mathrm{~d}$ eclipsing binary with similar mass stellar components $\left(M_{1}=\right.$ $\left.1.56 M_{\odot}, M_{2}=1.35 M_{\odot}\right)$. The stars are in a circular orbit, as exhibited by the sinusoidal shape of the RV and occurrence of secondary eclipse at a phase of $\sim 0.5$ as seen in Figure 3 .

Because the eclipses in the light curves are shallow (4\% loss of light), the impact parameter is highly degenerate with the flux and radius ratio. That is, a solution with similar flux contributions from both components in a more inclined system yields the same shallow eclipses as a solution with a much brighter primary component in a more edge-on system. This degeneracy can be ameliorated with additional information from spectra. Therefore, we place a Gaussian prior on the flux ratio parameter based on the BF fits, with a $0.21 \sigma$ width. With this flux ratio constraint, we find the secondary star is about $80 \%$ the size of the primary, with absolute dimensions of $2.0 R_{\odot}$ and $1.7 R_{\odot}$ for the primary and secondary components, respectively.

\section{KIC 5285607}
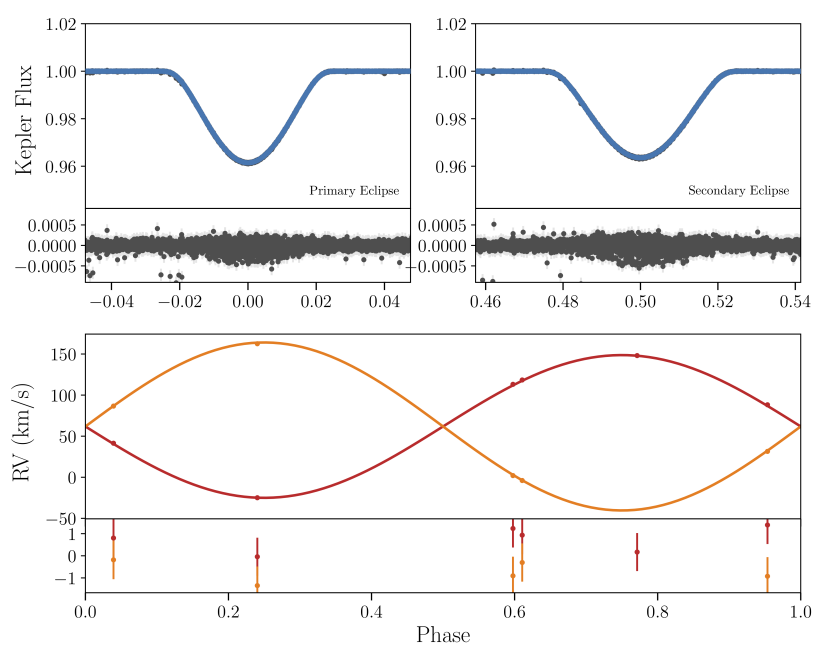

Figure 3. Simultaneous light curve (top panels) and radial velocity (bottom panel) fits to Kepler and APOGEE observations for KIC 5285607. The light curve data and residuals are shown in dark grey, with the model overlaid in blue. Note the shallow and V-shaped primary and secondary eclipses, which indicate a grazing system. The phased RV panel shows the BF derived values (points) and corresponding model (lines), where the primary RV is red and secondary $\mathrm{RV}$ is orange. The RV semi-amplitude is $\sim 100 \mathrm{~km} / \mathrm{s}$ while the residual scatter is $\sim 1 \mathrm{~km} \mathrm{~s}^{-1}$.
KIC 6864859 is a highly eccentric $(e \sim 0.6)$, slightly inclined $\left(i=88.32^{\circ}\right) 40.9 \mathrm{~d}$ eclipsing binary with components of similar mass $\left(M_{1}=1.41 M_{\odot}, M_{2}=1.35 M_{\odot}\right)$ and radii $\left(R_{1}=1.66 R_{\odot}, R_{2}=1.46 R_{\odot}\right)$. The best-fit model is shown in Figure 4 . The system's highly eccentric orbit gives rise to irregularly shaped RVs and a secondary eclipse near phase $\sim 0.125$.

Figure 5 shows clear brightening events of the system between primary and secondary eclipses, with maximal amplitudes $\sim 0.3 \mathrm{ppt}$ at phase $\sim 0.065$, the predicted phase of periastron passage from KEBLAT. This behaviour is consistent with tidal distortions in an eccentric orbit near periastron, and is symptomatic of a class of objects known as "heartbeat" stars (Thompson et al. 2012). Both primary and secondary eclipse residuals exhibit small amplitude ( $\sim 0.5 \mathrm{ppt})$ coherent structures; these are likely due to the non-spherical shape of the stars which is not explicitly modeled in KEBLAT.

We note that in our reduction process, some apVisit spectra were eliminated due to a very low signal-to-noise ratio that persisted after being run through our de-spike program. The majority of the remaining visits for this target resulted in well-separated peaks from the BF.

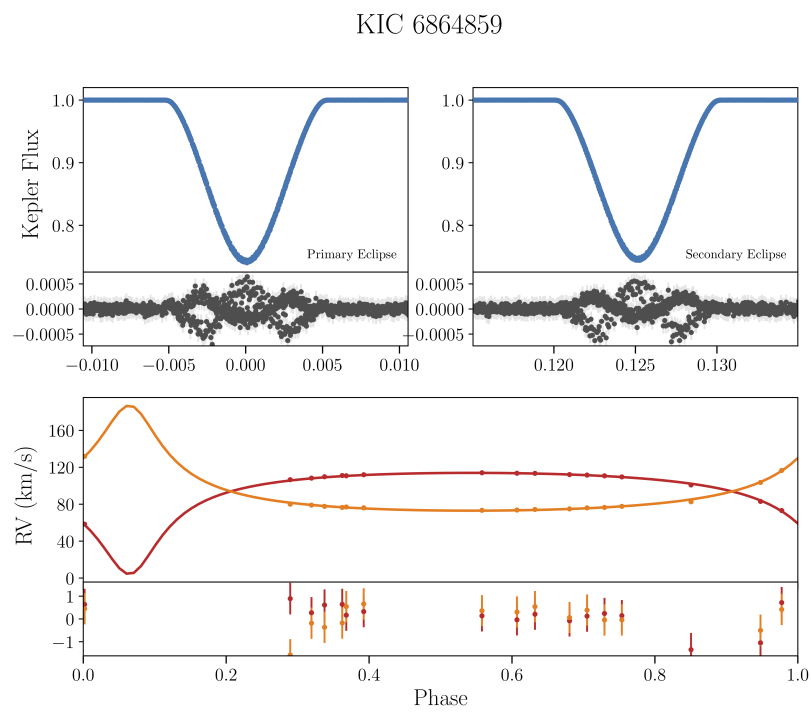

Figure 4. Simultaneous light curve (top panels) and radial velocity (bottom panel) fits to Kepler and APOGEE observations of KIC 6864859. The primary and secondary eclipses are similar in shape and depth with $\sim 25 \%$ loss of light; the phase of secondary eclipse and shapes of the RVs indicate an extremely eccentric system. The light curve residuals are small but have a coherent shape, likely due to tidal and rotational distortion of the stars. 


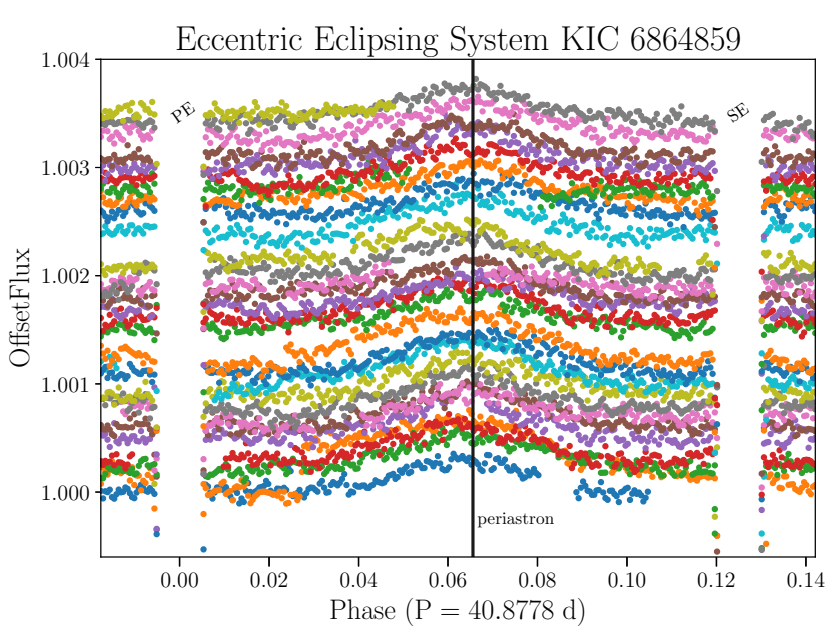

Figure 5. "Heartbeat" signatures of the eccentric eclipsing system KIC 6864659. The light curve surrounding primary and secondary eclipses is folded in phase and offset vertically between each observed orbit, using different colors for visual clarity. The brightening between primary and secondary eclipse is readily apparent around phase 0.065 .

KIC 6778289 is a $30.1 \mathrm{~d}$ eclipsing binary with stellar components $M_{1}=1.51 M_{\odot}$ and $M_{2}=1.09 M_{\odot}$ in an eccentric $(e=0.2)$, nearly edge-on $\left(i=89.3^{\circ}\right)$ orbit. The simultaneous $\mathrm{RV}+\mathrm{LC}$ fit is shown in Figure 6. The radii are $R_{1}=1.75 R_{\odot}$ and $R_{2}=1.0 R_{\odot}$. This difference in radius gives rise to the difference in primary and secondary eclipse shape. The flat-bottom secondary eclipse indicates a total eclipse of the secondary component, while the primary eclipse is more $\mathrm{V}$-shaped, e.g., more grazing. The larger residuals during secondary eclipse is consistent with starspot modulation. The system has low $(\approx 1 \%)$ third light contamination in the Kepler light curve which does not appear in the BF.

\subsection{KIC 6449358}

KIC 6449358 is a 5.8 d circular EB which may be a gravitationally bound to a distant tertiary companion.

The BF for this object exhibits two clear peaks, however, the second-brightest peak is relatively stationary in RV while the brighter peak varies by $\sim 60 \mathrm{~km} / \mathrm{s}$ over one orbit. This is shown in Figure 7 as well as in Appendix A Figure 19. If the $\sim$ stationary BF peak corresponded to a stellar binary component, it would require a system with extremely large mass ratio $M_{2} / M_{1}>10$, which would be consistent with a white dwarf. However, the light curve constrains the radius ratio to be $R_{2} / R_{1} \sim 0.3$, which makes this scenario physically implausible.

A more likely explanation for the $\sim$ stationary RV component is that it belongs to a tertiary star, and that the true secondary stellar component of the EB is too
KIC 6778289
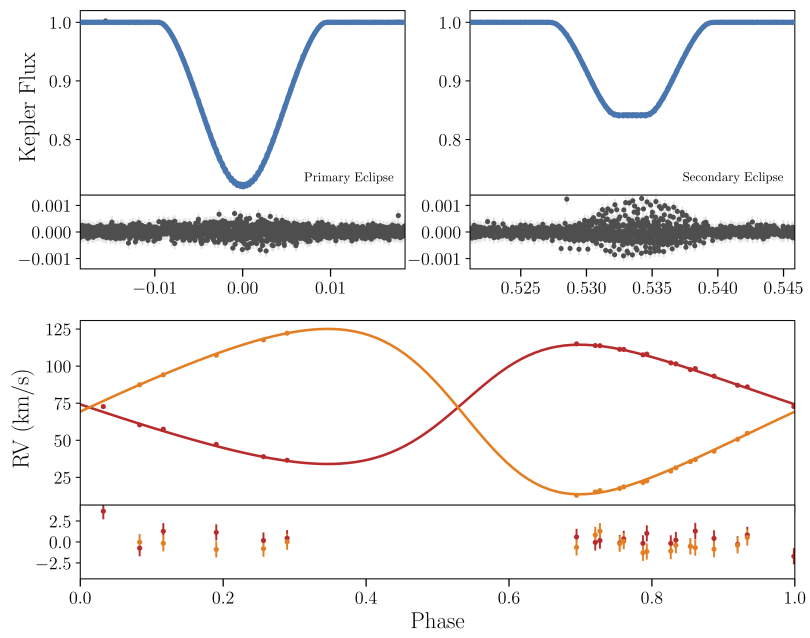

Figure 6. Simultaneous light curve (top panels) and radial velocity (bottom panel) fits to Kepler and APOGEE data for KIC 6778289. Different eclipse depths along with a flatbottomed secondary eclipse indicate a smaller and dimmer secondary. Additionally, the unequal amplitudes and shape of the RV indicates an unequal mass binary with significant orbital eccentricity.

faint to be robustly detected by APOGEE. Indeed, the flux ratio in the Kepler bandpass is $F_{2} / F_{1} \sim 0.01$. Thus, we effectively treat KIC 6449358 as a single-lined SEB in our model, and as a result we are only able to constrain the mass function $f_{M}$ of the binary. Specifically, to reproduce the observed RV amplitude, our sum and ratio of masses solutions are degenerate, tending toward two extremes: high total mass $\left(\sim 4 M_{\odot}\right)$ with a low mass ratio $(q \sim 0.3)$, or low total mass $\left(1 M_{\odot}\right)$ with a higher mass ratio $(q \sim 0.67)$.

We note that some APOGEE visits do suggest a small, third BF peak (see Appendix A Figure 20 for details). These marginal $\mathrm{BF}$ peaks have large radial velocity variations from visit to visit, consistent with a low-mass star. This supports the scenario with total mass $\sim 2.3 M_{\odot}$ and mass ratio $q \sim 0.45$.

Figure 7 shows the best KEBLAT model fit to the light curve and radial velocities obtained using mass function $f_{M}=\frac{M_{2}^{3} \sin i}{\left.M_{1}+M_{2}\right)^{2}}$ her than $M_{1}$ and $M_{2}$. The $\sim$ stationary RV points, which are not fit, correspond to the putative third star, either a line-of-sight coincident or a tertiary companion in a hierarchical triple system. We favour the latter scenario, as the eclipses show timing variations consistent with perturbations by a bound, tertiary component. These eclipse timing variations (ETVs) have been used to identify and characterize many Kepler EBs (e.g. Borkovits et al. 2016), and we fit these ETVs using 
a simple linear ephemeris based on the observed times of primary eclipse. We then compute the observed minus computed $(O-C)$ eclipse times as a function of time. The result is shown in Figure 8. There is a clear parabolic or sinusoidal trend in the ETVs with an amplitude of $\sim 0.0006 \mathrm{~d}$; the ETV timescale indicates that the perturbing tertiary body has a minimum period $\sim 1450$ d. The architecture of this type of hierarchical triple short, circular inner binary orbited by a distance tertiary companion - is consistent with dynamical processing via the Kozai (1962) mechanism.

\section{KIC 6449358}
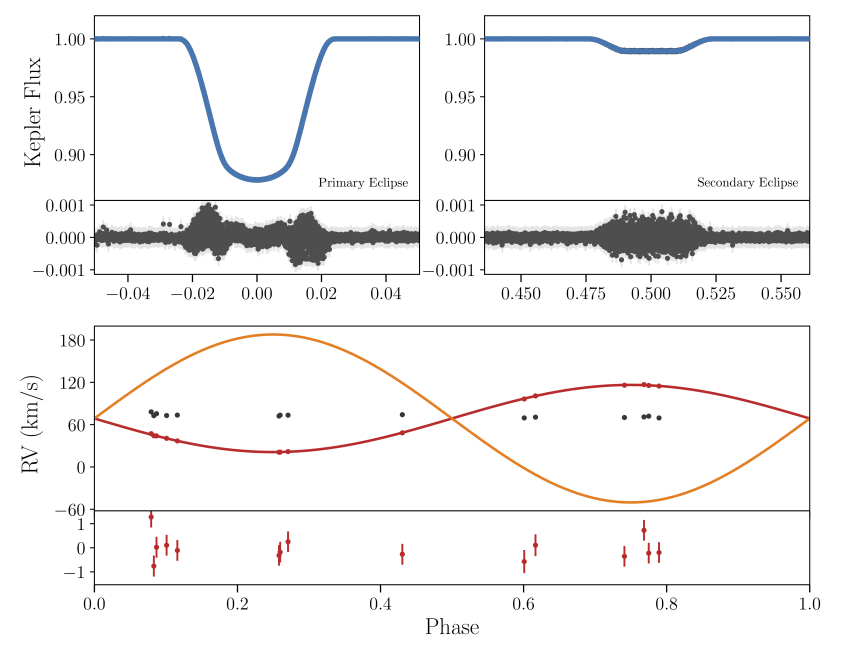

Figure 7. Simultaneous RV+LC fit for KIC 6449358, a single-lined spectroscopic binary suspected in a hierarchical triple system. We utilize mass function her than individual component masses to obtain the RV fit. The stationary RV points near $\sim 80 \mathrm{~km} / \mathrm{s}$ are measured RVs of a tertiary companion, while the orange curve shown here is the predicted radial velocity of the unseen secondary, based on the locations of tentative BF peaks in Appendix A Figure 20. The flat-bottom secondary eclipse indicates the system is close to edge-on with a radius ratio $\sim 0.33$, breaking the inclinationradius ratio degeneracy. The primary eclipse residuals are significant during ingress and egress, consistent with eclipse timing variations due to a tertiary companion (see Figure 8).

\section{5. $K I C 4285087$}

KIC 4285087 is an equal mass binary $\left(M_{1} \approx M_{2} \approx\right.$ $\left.1.1 M_{\odot}\right)$ in a circular, slightly inclined $\left(i=87.3^{\circ}\right) 4.5 \mathrm{~d}$ orbit. We show the best-fit solution in Figure 9. The components are main-sequence dwarfs with similar radii $\left(R_{1} \sim R_{2} \sim 1 R_{\odot}\right)$. The eclipses are similar depth $(\sim$ $30 \%$ ), duration $(\sim 0.2 \mathrm{~d})$, and shape $(\mathrm{V})$, consistent with equal mass dwarfs orbiting each other.

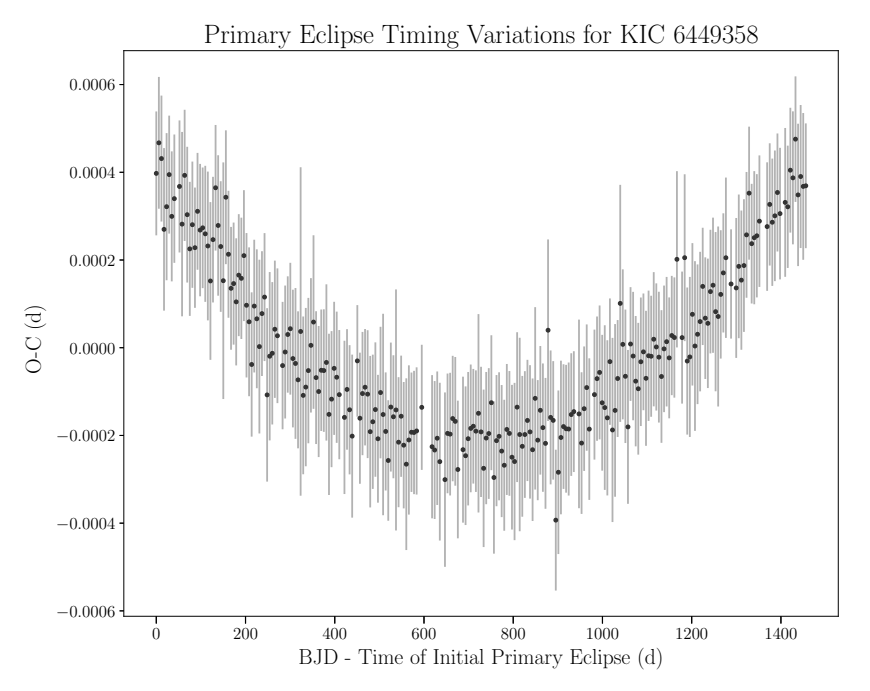

Figure 8. O-C diagram for KIC 6449358, showing primary eclipse timing variations; the ETVs exhibit a half-sinusoid trend, giving a rough estimate for the minimum period of the tertiary perturber to be $P \sim 1450 \mathrm{~d}$.

KIC 4285087
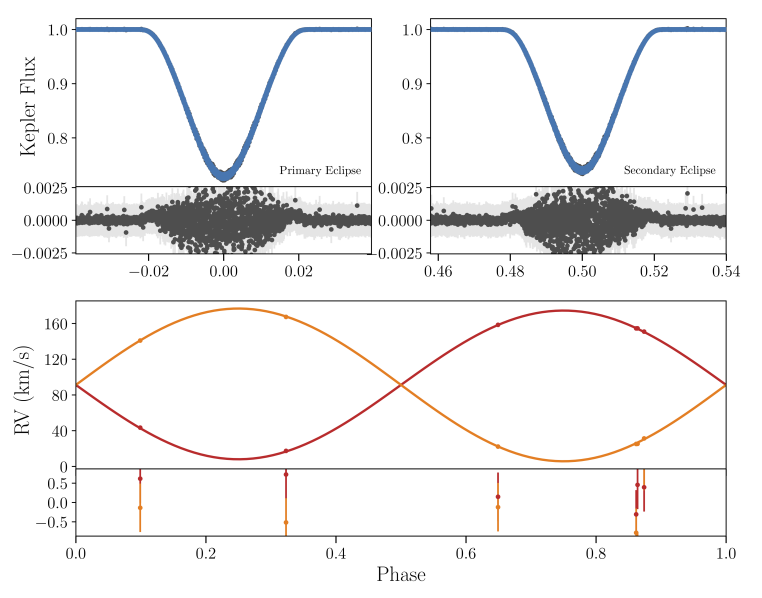

Figure 9. Simultaneous light curve (top panels) and radial velocity (bottom panel) fits to Kepler and APOGEE observations. The phase of secondary eclipse and shape of RVs indicate EBs in a circular orbit. The light curve residuals during eclipse suggest the presence of a variable third light contribution, or non-Keplerian photometric effects given its short period. $(\sim 4 \mathrm{~d})$.

\subsection{KIC 6131659}

KIC 6131659 is a mass ratio of 0.75 binary $\left(M_{1}=\right.$ $\left.0.9 M_{\odot}, M_{2}=0.7 M_{\odot}\right)$ in a $17.5 \mathrm{~d}$, close-to-circular orbit. Figure 10 shows the simultaneous RV + LC fit to the data. The primary and secondary eclipses are relatively 
deep, with $35 \%$ and $10 \%$ loss of total system light, respectively. The residuals to the light curve fit show correlated structure, which may be due to poor limb darkening modeling and/or a varying third light component which deviates from Kepler crowding values.

There is a third light component readily visible in the BF (see Appendix A Figure 22, but it is not RVvariable. This suggests it may be a line-of-sight contamination source or a gravitationally bound body in a hierarchical triple with an orbital period much longer than $17.5 \mathrm{~d}$. The light curve does not show apparent eclipse timing variations, but this does not preclude the presence of a gravitationally bound tertiary.

\section{KIC 6131659}
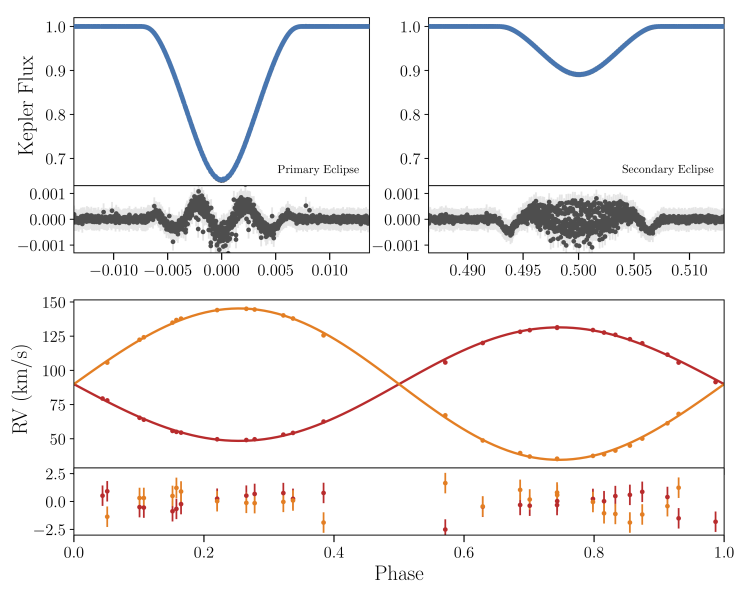

Figure 10. Simultaneous light curve (top panels) and radial velocity (bottom panel) fits to Kepler and APOGEE observations for KIC 6131659. The primary and secondary eclipses are relatively deep, with $35 \%$ and $10 \%$ loss of total system light, respectively.

\section{7. $K I C 6781535$}

KIC 6781535 is an eccentric $(e=0.25)$, grazing $\left(i=84^{\circ}\right), 9.1 \mathrm{~d}$ binary. The best-fit solution (see Figure 11) yields binary components of similar mass $\left(M_{2} / M_{1} \approx\right.$ $1.0)$ but slightly different radii $\left(R_{2} / R_{1} \approx 1.2 \pm 0.1\right)$, which suggests a slightly evolved system. The shallow eclipses poorly constrain the system's impact parameter, flux ratio, and radius ratio. As a result of this degeneracy, we apply a Gaussian prior on the light curve flux ratio parameter from spectra, following the same method as used for KIC 5285607 (see 3.1 ).

Similarly to KIC 6131659 , there is a third light component visible in the BF (see Figure 23) that is not RV variable, indicating either a line-of-sight coincident third star or gravitationally bound tertiary companion. There are symmetric structures in the light curve residuals, most noticeably during primary eclipse, consistent with variable third light contribution, changes to the binary orbit due to additional bodies, or starspot modulations. Because the system exhibits shallow, grazing eclipses, it was not conducive to an ETV analysis.

\section{KIC 6781535}
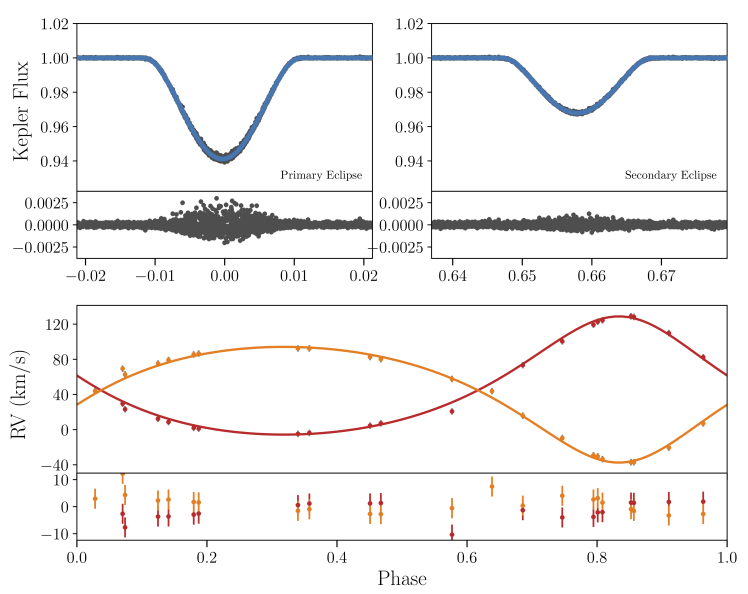

Figure 11. Top panels show the light curve model (blue) for KIC 6781535 overlaid against data (grey) as a function of phase. Bottom panel shows the RV model (lines) for primary (red) and secondary (orange) components overlaid on top of APOGEE-extracted data (points). The models correspond to the best-fit joint LC+RV solution.

\subsection{Supplemental Physical Parameters}

In addition to the main results in Table 3 we report some additional physical parameters in Table 3 As discussed in Section 2.6. we can use the BF peak area ratios to measure the $H$-band flux ratio of each system. We can also combine Gaia parallax information with our measured fluxes, radii, and the ASPCAP $T_{\text {eff }}$ to estimate individual stellar temperatures. These parameters, along with adopted values from external sources, are summarized in Table 3 ,

\section{DISCUSSION}

\subsection{Evolutionary Histories}

With such well-characterized stars, we can investigate each binary's age and evolutionary history with two different approaches. In the following we assume normal Milky Way metallicities $-0.5<[\mathrm{M} / \mathrm{H}]<0$.

First we explore the H-R diagram in $\log g$ vs. $\log T_{\text {eff }}$ after first correcting our temperature estimates following El-Badry et al. (2017). We calculate $\log g$ for each star directly from the KEBLAT mass and radius. We also 
Table 3. Parameters related to RV extraction and temperature estimates

\begin{tabular}{lccccccc}
\hline \hline & KIC 5285607 & KIC 6864859 & KIC 6778289 & KIC 6449358 & KIC 4285087 & KIC 6131659 & KIC 6781535 \\
\hline BF Flux Ratio $\left(F_{2} / F_{1}\right)$ & $0.620 \pm 0.027$ & $0.811 \pm 0.028$ & $0.462 \pm 0.029$ & $0.392 \pm 0.014$ & $0.997 \pm 0.023$ & $0.648 \pm 0.028$ & $1.253 \pm 0.115$ \\
ASPCAP $T_{\text {eff }}(\mathrm{K})$ & $6495 \pm 156$ & $6417 \pm 159$ & $6572 \pm 162$ & $6237 \pm 179$ & $5664 \pm 146$ & $4845 \pm 98$ & $5749 \pm 125$ \\
Gaia parallax (mas) $b$ & $1.254 \pm 0.0216$ & $1.4897 \pm 0.0241$ & $0.9093 \pm 0.0222$ & $1.1974 \pm 0.0264$ & $1.619 \pm 0.0312$ & $-0.5117 \pm 1.0713$ & $\ldots$ \\
Gaia distance (pc) $b$ & $799 \pm 14$ & $671 \pm 11$ & $1100 \pm 27$ & $835 \pm 18$ & $617 \pm 12$ & $\ldots$ & $\ldots$ \\
$\log g_{1}(\mathrm{cgs})$ & $4.028 \pm 0.013$ & $4.150 \pm 0.010$ & $4.133 \pm 0.008$ & $\ldots$ & $4.454 \pm 0.006$ & $4.496 \pm 0.005$ & $4.161 \pm 0.019$ \\
$\log g_{2}(\mathrm{cgs})$ & $4.118 \pm 0.015$ & $4.244 \pm 0.012$ & $4.479 \pm 0.010$ & $\ldots$ & $4.478 \pm 0.007$ & $4.705 \pm 0.006$ & $4.253 \pm 0.021$ \\
$T_{\text {eff }}$ offset $(\mathrm{K})$ & 350 & 80 & 250 & $\ldots$ & 20 & 350 \\
Adopted $T_{\text {eff, }, 1}(\mathrm{~K})$ & $6845 \pm 328$ & $6497 \pm 159$ & $6822 \pm 162$ & $6737 \pm 178$ & $5689 \pm 146$ & $5195 \pm 98$ & $5849 \pm 125$ \\
Adopted $T_{\text {eff, }, 2}(\mathrm{~K})$ & $6716 \pm 293$ & $6541 \pm 283$ & $7265 \pm 440$ & $8788 \pm 658$ & $5735 \pm 105$ & $\ldots$ & $\ldots$ \\
\hline
\end{tabular}

${ }^{a}$ DR14 (Pérez et al. 2016)

$b$ Bailer-Jones et al. 2018

${ }^{c}$ Computed directly from $M$ and $R$ as reported in Table 3

$d$ El-Badry et al. (2017)

use the KEBLAT masses and radii to determine the system ages in the mass-radius space directly, which avoids any dependence on distance or on our disentangling of the individual component temperatures.

In both approaches, we use Dartmouth evolutionary tracks (Dotter et al. 2008) and consider only the portion of the track with $\log g \geq 4$.1. This effectively only includes the main sequence. For consistency, we adhere to the KEBLAT definition of star 1 (primary) and star 2 (secondary) in which star 1 is the member of the SEB being eclipsed during the primary eclipsing event, and star 2 (secondary) as the member eclipsed during the secondary eclipsing event.

Figure 12 shows all of the SEBs in the $\log g$ vs. $\log T_{\text {eff }}$ diagram, and Figure 13 shows each SEB system individually. In general, the systems are broadly consistent with ages ranging from about 0.8 to about $3 \mathrm{Gyr}$, and the two components of each system appear to be consistent with a common age. Figure 14 represents these systems in the mass-radius diagram, where again all six systems modeled appear consistent with coevality for the same range of ages as above.

\subsection{Mass-Luminosity Relationships}

In order to verify that our targets are on the main sequence, we create a mass-luminosity plot (Figure 15 ) using the stellar masses from Table 3 and calculated $H$-band luminosities explained below. These luminosities are independent of the ASPCAP temperature estimates and corrections from El-Badry et al. (2017) used to derive Figures 13 and 14 . The results represent a

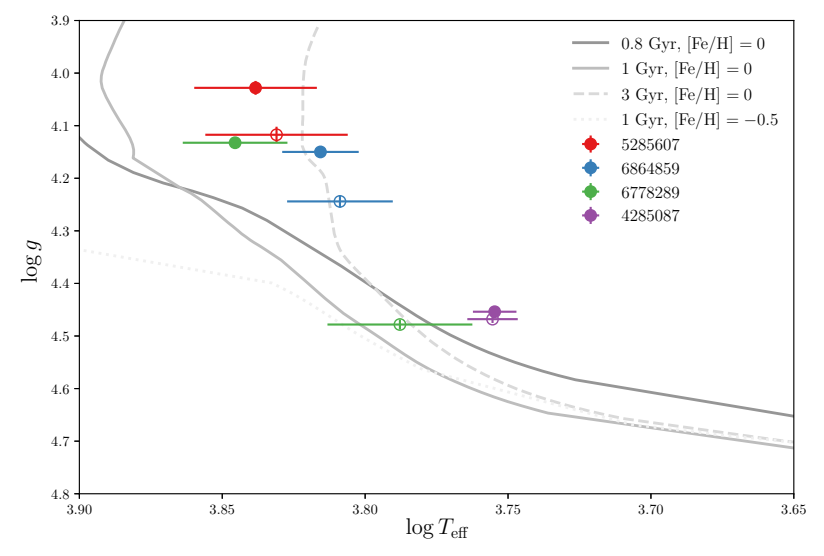

Figure 12. Spectroscopic H-R diagram for the systems with Gaia distances. Primaries are depicted with the solid circles while secondaries are open circles. A variety of Dartmouth isochrones are plotted with a range of ages 0.8-3 Gyr and metallicities (sub-solar to solar). Only the four targets with Gaia parallaxes are plotted here. Assuming Milky Way metallicity, we find all of our systems exhibit a high degree of coevality with ages ranging from 1-3 Gyr.

comparison to theoretical models that complements the mass-radius relationship presented in Figure 14, and is less reliant on light curve modeling, which may have degenerate radius solutions in grazing geometries.

To calculate $H$-band luminosities, we use distances derived from Gaia parallaxes (Bailer-Jones et al. 2018) to convert apparent $H$-band magnitudes from 2MASS (Skrutskie et al. 2006) to absolute magnitudes. We 


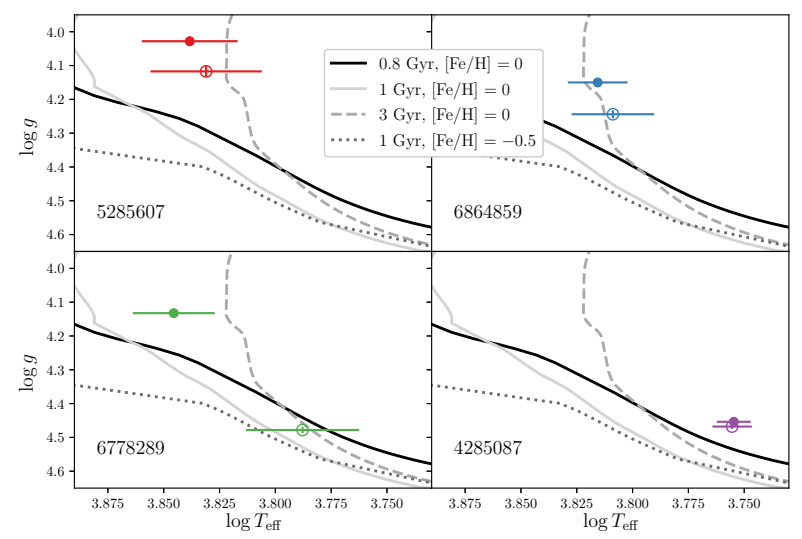

Figure 13. Figure 12 showing each SEB system individually. The filled circles model the primary star of the SEB, and the hollow circles the secondaries.

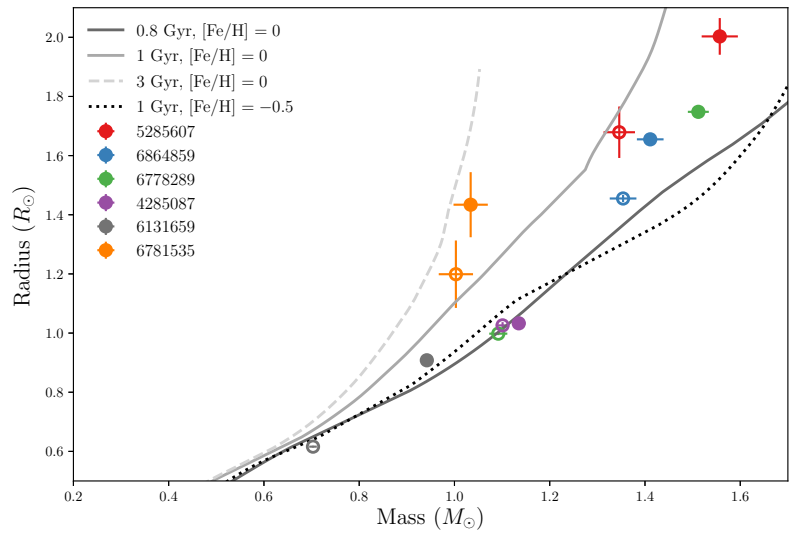

Figure 14. Radius versus mass diagram for all seven systems. Primaries are shown as solid circles and secondaries in open circles. Dartmouth isochrones for a variety of ages and metallicities are also plotted. All seven systems are consistent with coevality, ranging in age from about 1 to about 3 Gyr, assuming a normal Milky Way metallicity of $[\mathrm{M} / \mathrm{H}]$ $=-0.5$

check that the $H$-band magnitudes were not taken during eclipse by cross referencing the time of 2MASS observations to the EB ephemeris. We then compute the system $H$-band luminosities from absolute magnitudes using the sun as a reference, with $H$-band magnitude of 3.32 from Cohen et al. (2003). We disentangle the separate luminosities for each stellar component in the system using the observed APOGEE $H$-band flux ratios (see Table 3). In Figure 15, we show each system using the same plotting convention as Figures 12 to 14 . where solid and open circles correspond to primary and secondary components, respectively. We over-plot for comparison theoretical masses and $H$-band magnitudes from Dartmouth isochrones (Dotter et al. 2008) at subsolar and solar metallicity and a range of ages $(0.8-5$ Gyr). In general, as previously concluded, members of the same binary system follow the same evolutionary track, i.e., are coeval.

We exclude KIC 6449398 from this analysis, because it is a single-lined SEB. We also exclude KICs 6131659 and 6781535, which have negative parallax values from Gaia. For this reason we could not include these targets in the spectroscopic H-R diagram for our systems with Gaia distances. We did not include reddening corrections to the distance modulus calculations; however, reddening effects should be minimal in the near-IR (2MASS and APOGEE $H$-band) compared to the visible (Kepler).

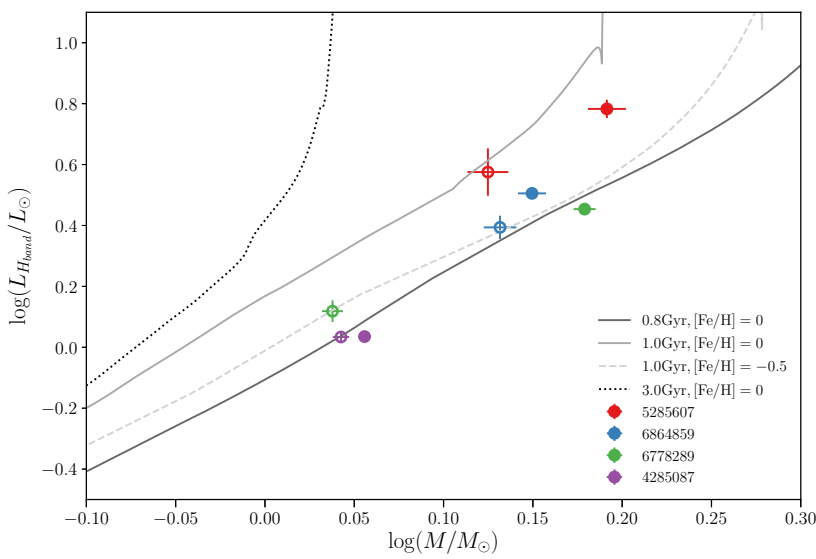

Figure 15. Mass-luminosity relationship for the four targets with accurate Gaia distances and RV-derived masses. Primaries are shown in solid circles and secondaries in open circles. Dartmouth isochrones (Dotter et al. 2008) are overplotted as grey lines, corresponding to a variety of ages and metallicities.

\subsection{Tertiary Companions}

In our subset of SEBs, we identified three candidate triple systems. The binaries with possible tertiary companions are KIC 6131659, KIC 6781535, and KIC 6449358 (see Sections 3.6, 3.7, and 3.4, respectively). The first two of these systems exhibit clear third BF component peaks in nearly all RV visits. We fit these third peaks with Gaussians, similar to the Gaussian fits for the primary and secondary BF components. In both cases, the third BF components do not have radial velocity variations above the noise, which suggest these third members are either line-of-sight contamination sources 
or gravitationally bound in hierarchical triples with orbital periods much longer than that of the observed SEB.

While statistics on multiplicity are not complete, numerous studies have found tertiary systems composed of a tight binary orbited by a distant third member. Statistics from Tokovinin et al. 2006) indicate that roughly $63 \%$ of spectroscopic binaries have tertiary companions in a wide orbit. In binaries with shorter periods (less than 3 days) this percentage rises to $96 \%$, but in longer period binaries (12 or more days) this percentage is only $34 \%$.

The incidence of triples among Kepler close eclipsing binaries (as are the ones in our analysis) is at least $\sim 20 \%$ (Rappaport et al. 2013; Conroy et al. 2014), and likely higher for tertiaries with longer periods beyond Kepler's finite observing time.

Evidence from both spectroscopic and photometric observations indicate KIC 6449358 belongs to a hierarchical triple system (see 33.4. The BF for this system shows a stationary tertiary peak in a few of the APOGEE visits. The mid times of eclipses in the $\mathrm{Ke}$ pler light curve also exhibit sinusoidal variations in time; these eclipse timing variations indicate that the tertiary has a period $\gtrsim 1450 \mathrm{~d}$.

Interestingly, among our sample we do not detect tertiary companions among the shortest-period binaries. In particular, neither of the two EBs with $P_{\text {orb }}<5 \mathrm{~d}$ exhibits a clear tertiary in our data. It is not yet clear whether existing observations might already exclude the presence of tertiaries at very large separations that might not appear in our data; additional imaging observations might be required to identify such stars. At the same time, two of the four EBs with $P_{\text {orb }}>9 \mathrm{~d}$ are triples, which would appear to be an over-abundance of tertiaries among the longest-period EBs, albeit with a small sample. However, we note that one of these (KIC $6781535, P_{\text {orb }} \approx 9 \mathrm{~d}$ ) is a modestly evolved system (see Figure 14, and excluding that case yields an occurrence of $1 / 3$ triples among our EBs with $P_{\text {orb }}>12 \mathrm{~d}$ ), fully consistent with the results of Tokovinin et al. (2006).

\section{SUMMARY}

We thoroughly characterize seven SEBs that have been observed by both Kepler and APOGEE. Our targets are selected from the Kepler EB catalog, and limited to bright, detached EB targets with both primary and secondary eclipses observed by Kepler, high inclination, and multiple APOGEE visits. We identify an additional 26 SEBs which may warrant similar studies. We demonstrate that the $\mathrm{BF}$ is a superior method for extracting RVs from APOGEE visit spectra compared to the CCF used in the present reduction pipeline. This is particularly true for systems with multiple RV variable components. While such an analysis is beyond the scope of this work, if the BF method were applied to the full data set of multiple-visit APOGEE targets, it would most likely reveal many previously unknown SEBs and other interesting RV-variable sources.

RVs are extracted from apVisit spectra using the BF method and the Kepler light curves are normalized, deweighted and modeled using KEBLAT. The light curve and RV solutions are first determined individually, and then computed simultaneously. We use the resulting physical parameters to estimate stellar temperatures, investigate coevality, and explore candidate triple systems.

Using our analysis we find our target's binary members are coeval with ages ranging from 1 to $3 \mathrm{Gyr}$, assuming normal Milky Way metallicity $(-0.5<[M / H]<$ $0)$. The exception is KIC 6781535 which lies closer to a slightly metal poor $([M / H] \sim-1.0) 3$ Gyr isochrone. Our systems being broadly consistent with coevality confirms a common assumption in star formation that members of multiples form at the same time, and also effectively calibrates stellar evolution modeling.

Overlap between large scale surveys like APOGEE, Kepler, and Gaia allows us to discover and analyze many diverse SEBs, including systems with very low flux ratios and those in higher order systems. The statistics on the triples within our subset with respect to the orbital period of inner binaries is broadly consistent with statistics from the field Tokovinin (1997), though there may be some tension with our sample in that the shortest-period EBs do not appear to be spectroscopic triples. This is in contrast to the expectation that shortest-period EBs are most likely to be hierarchical triples. It is possible that very wide tertiaries do exist in these systems but have yet to be identified via imaging.

We have shown that through tools like KEBLAT and the BF analysis of APOGEE spectra, it is possible to perform high quality analysis of large numbers of SEBs with a variety of properties. This opens up great promise for future SEBs identified in TESS and SDSS-V data. 


\section{ACKNOWLEDGMENTS}

We would like to thank Scott Fleming for critical guidance and discussion and Paul A. Mason for valuable brainstorming and advice. We recognize the SDSS Faculty And Student Team (FAST) initiative for supporting this work through a partnership with New Mexico State University. JMCC thanks the Fisk-Vanderbilt Masters-to-PhD Bridge Program, Amanda Cobb, Kelly Holley-Bockelmann, and Nancy Chanover for continued empowerment of a woman and new mother in STEM. MLR celebrates that this work has encompassed two births, one wedding, and multiple graduations among the lead authors.

Software: Astropy Astropy Collaboration et al. 2013, Price-Whelan et al. 2018), PyAstronomy (Czesla
2018), Matplotlib (Hunter 2007), Apogee (Bovy|2016), Makecite (Price-Whelan et al. 2018), Numpy (Van Der Walt et al. 2011), Pandas (McKinney 2010), Emcee (Foreman-Mackey et al. 2013), Gaussfitter (Ginsburg 2017), Scipy (Jones et al. 2001)

\section{APPENDIX}

\section{A. STELLAR RADIAL VELOCITIES}

Here, we include one Broadening Function plot for each of the seen targets. These illustrate how we measured radial velocities for each component of the spectroscopic binaries from APOGEE visit spectra, as discussed in Section 2.2 . Note each figure uses uncorrected RV on the abscissa, before barycentric corrections have been applied. The final corrected RVs with uncertainties are reported in Table 3 below.

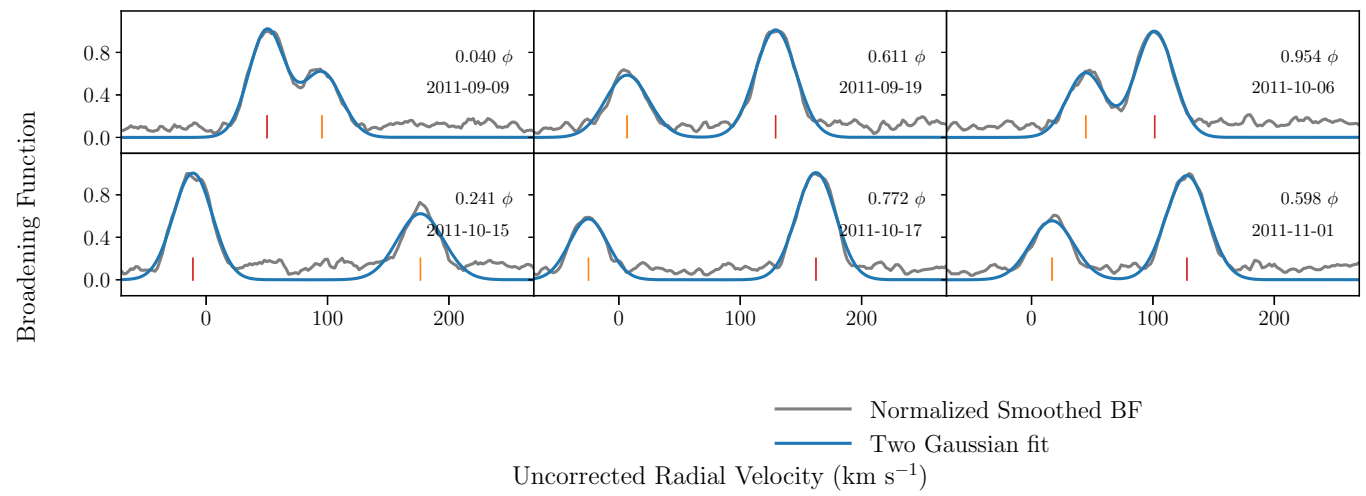

Figure 16. BF plots for KIC 5285607. The normalized smoothed BF is shown in grey while the Gaussian fits are modeled in blue. Uncorrected radial velocities are shown on the abscissa in $\mathrm{km} \mathrm{s}^{-1}$, and arbitrary amplitude of the BF on the vertical axis. In this case the primary (red) is distinguishable from the secondary (orange), and the visit spectra were well separated over the coarse of the observations. 


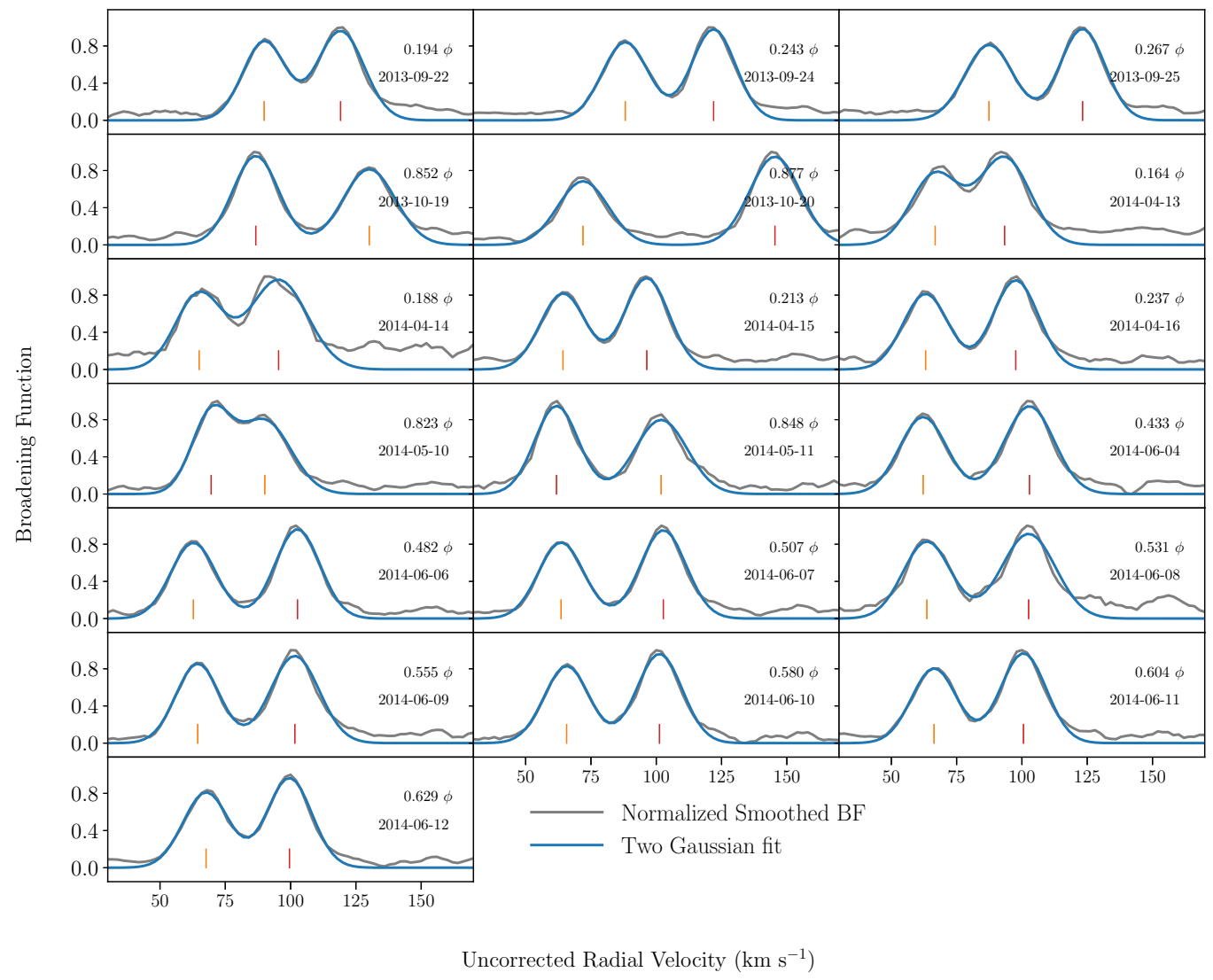

Figure 17. Same as Figure 16 but for KIC 6864859. In this case the primary (red) and secondary (orange) are less distinguishable due to the greater radius of the primary. Observations often were within a day of one another in their respective visit sets. Some visits were removed due to the presence of noise that could not be eliminated with our despiking method. 


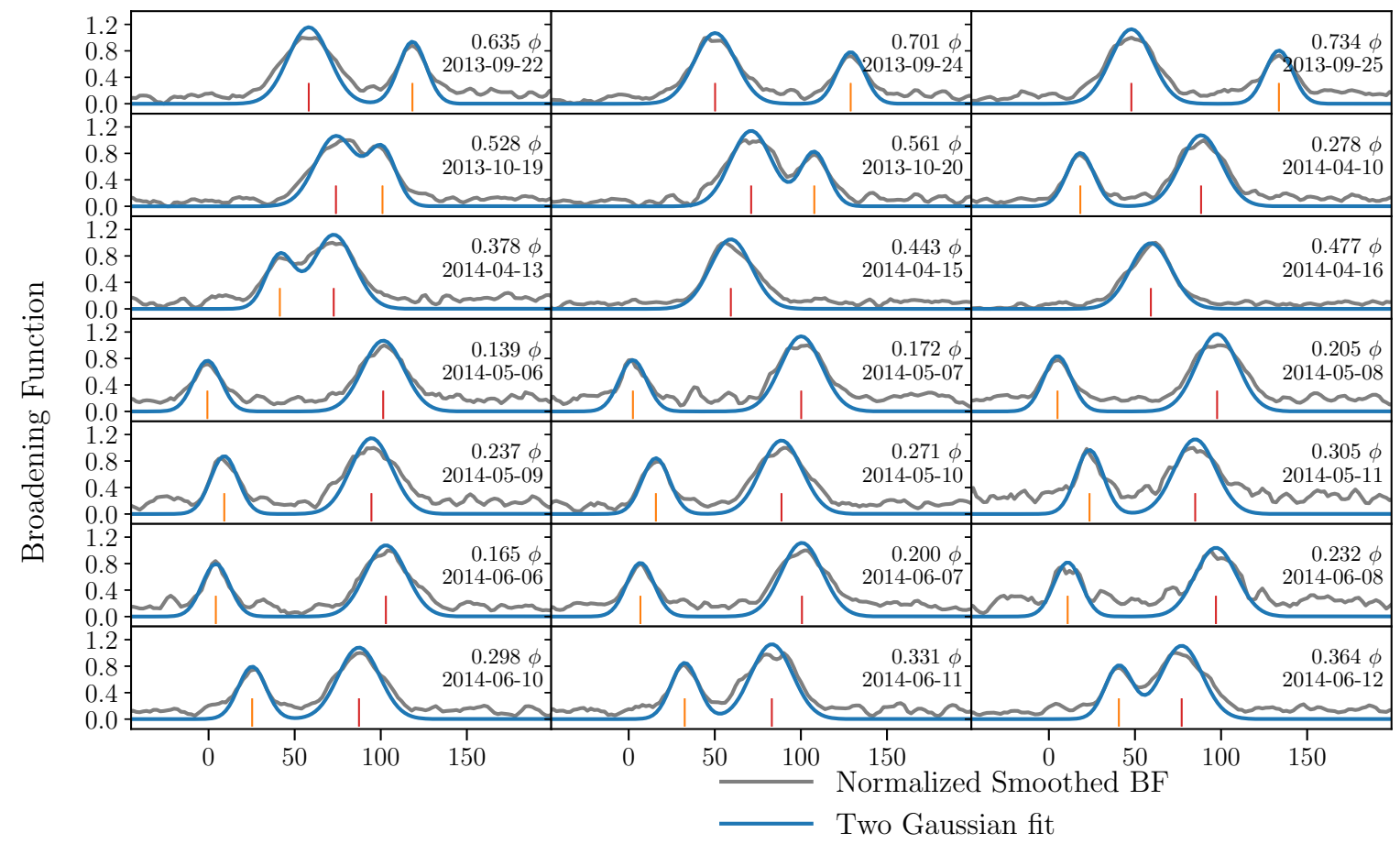

Uncorrected Radial Velocity $\left(\mathrm{km} \mathrm{s}^{-1}\right)$

Figure 18. Same as Figure 16 but for KIC 6778289. In this case the primary (red) is slightly distinguishable from the secondary (orange), and the visit spectra were well separated over the coarse of the observations. Some visits were removed due to excess noise after having been ran through our despiking method. 


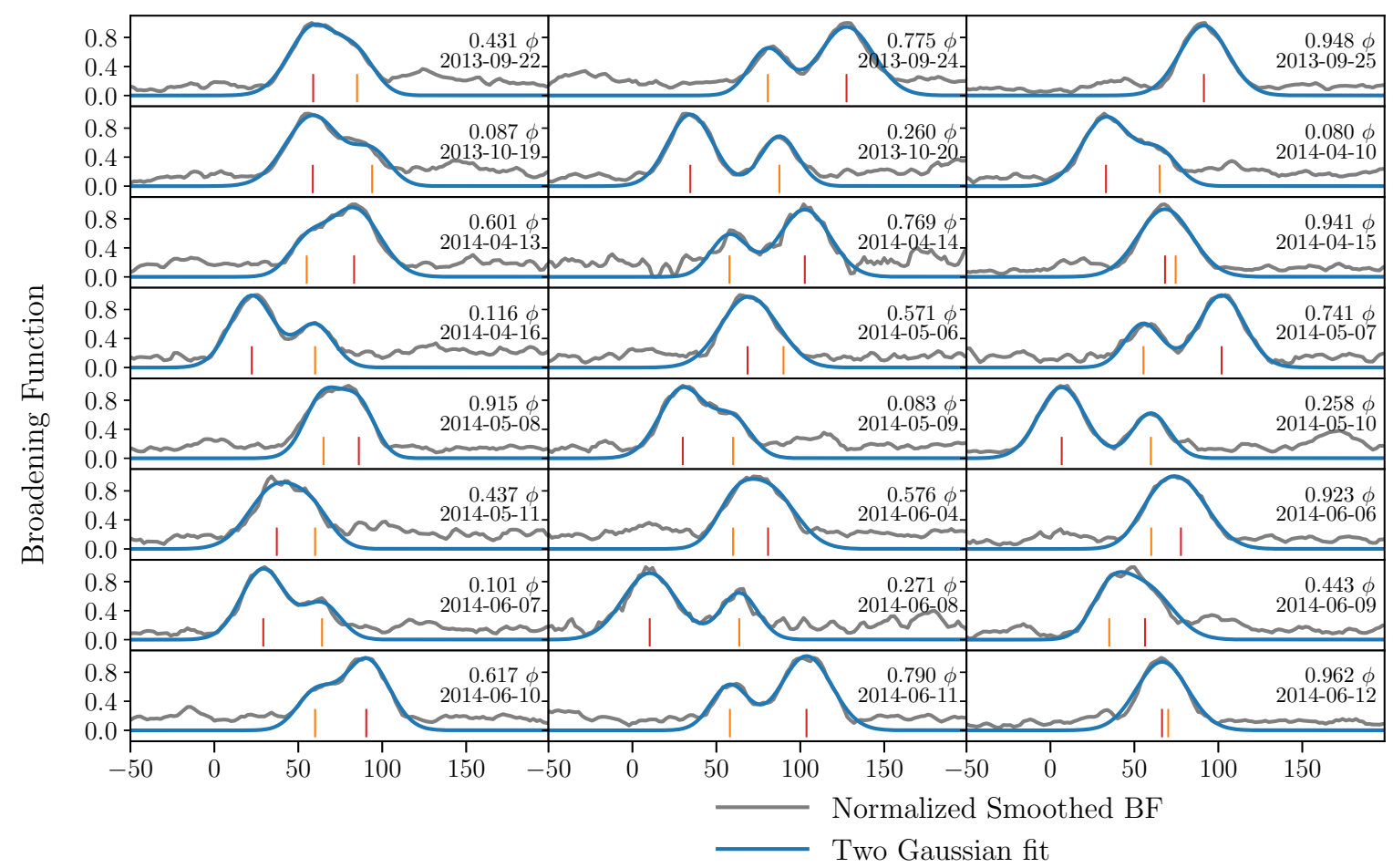

Uncorrected Radial Velocity $\left(\mathrm{km} \mathrm{s}^{-1}\right)$

Figure 19. Same as Figure 16 but for KIC 6449358. In this case the primary (red) is easily distinguishable from the secondary (orange). This target had visits in which the primary and secondary were very close together but not directly on top of one another, in these occurrences error is more pronounced in the radial velocities extracted. 

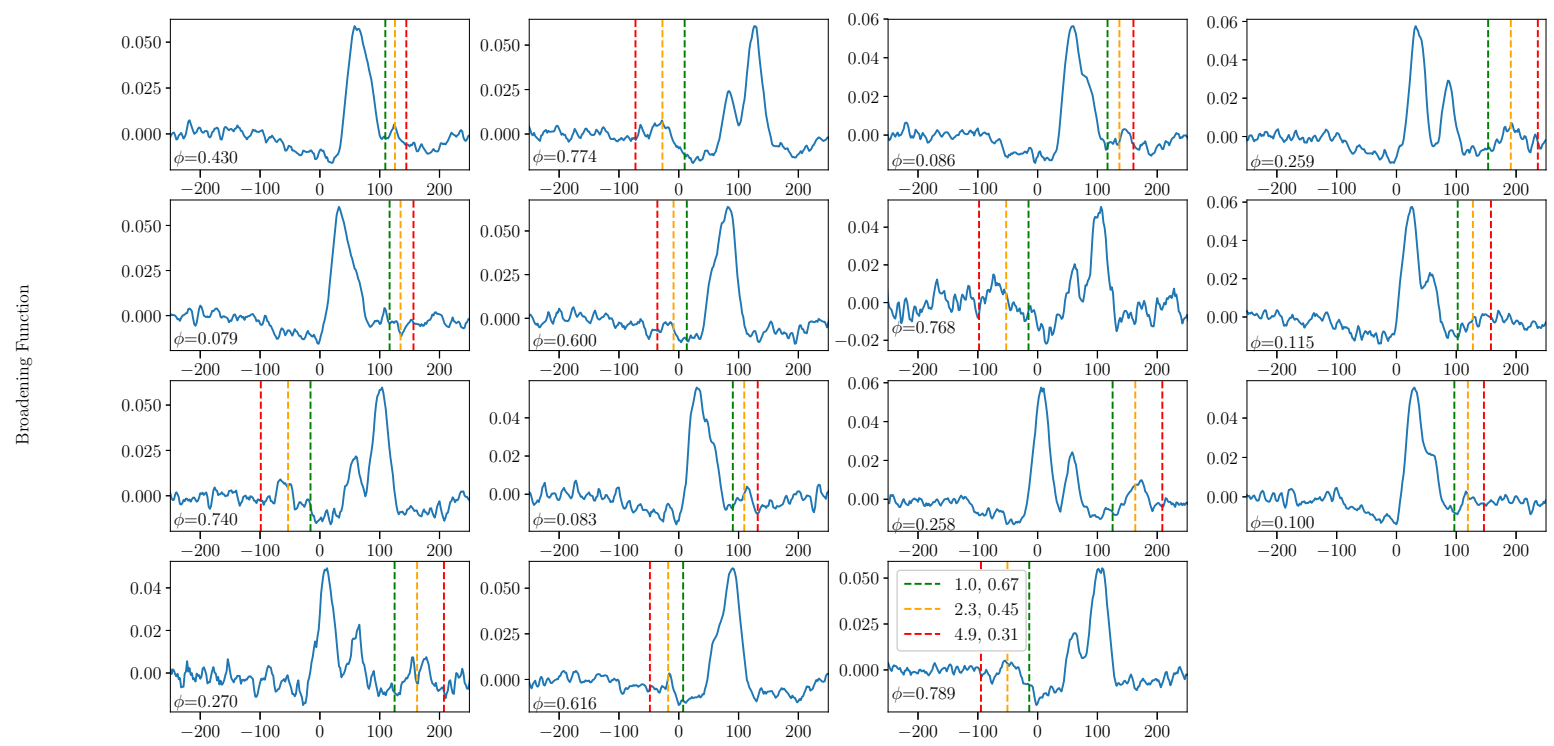

Figure 20. BF (blue solid line; zoomed out in each panel to $\pm 250 \mathrm{~km} \mathrm{~s}^{-1}$ ) of KIC 6449358 overplotted with expected locations of the secondary component's BF peak corresponding to various $M_{1}+M_{2}, M_{2} / M_{1}$ combinations (green, orange, red dashed lines). In a few of the visits, tentative BF peaks coincide with the secondary's expected locations for $M_{1}+M_{2} \sim 2.3, M_{2} / M_{1} \sim 0.45$ (see, e.g., panels corresponding to phase $=0.741,0.083,0.258,0.259,0.430$ ). In general, however, additional BF "peaks" are lost in the noise of the spectra.

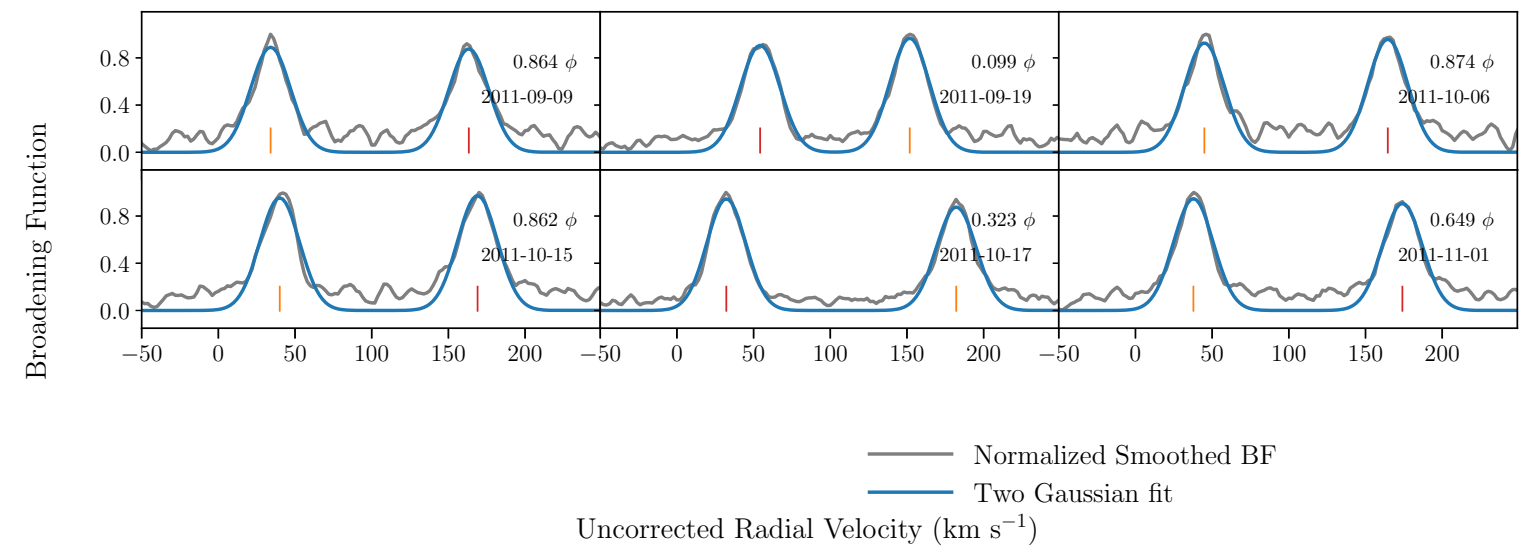

Figure 21. Same as Figure 16 but for KIC 4285087. In this case the primary (red) is easily distinguishable from the secondary (orange). 


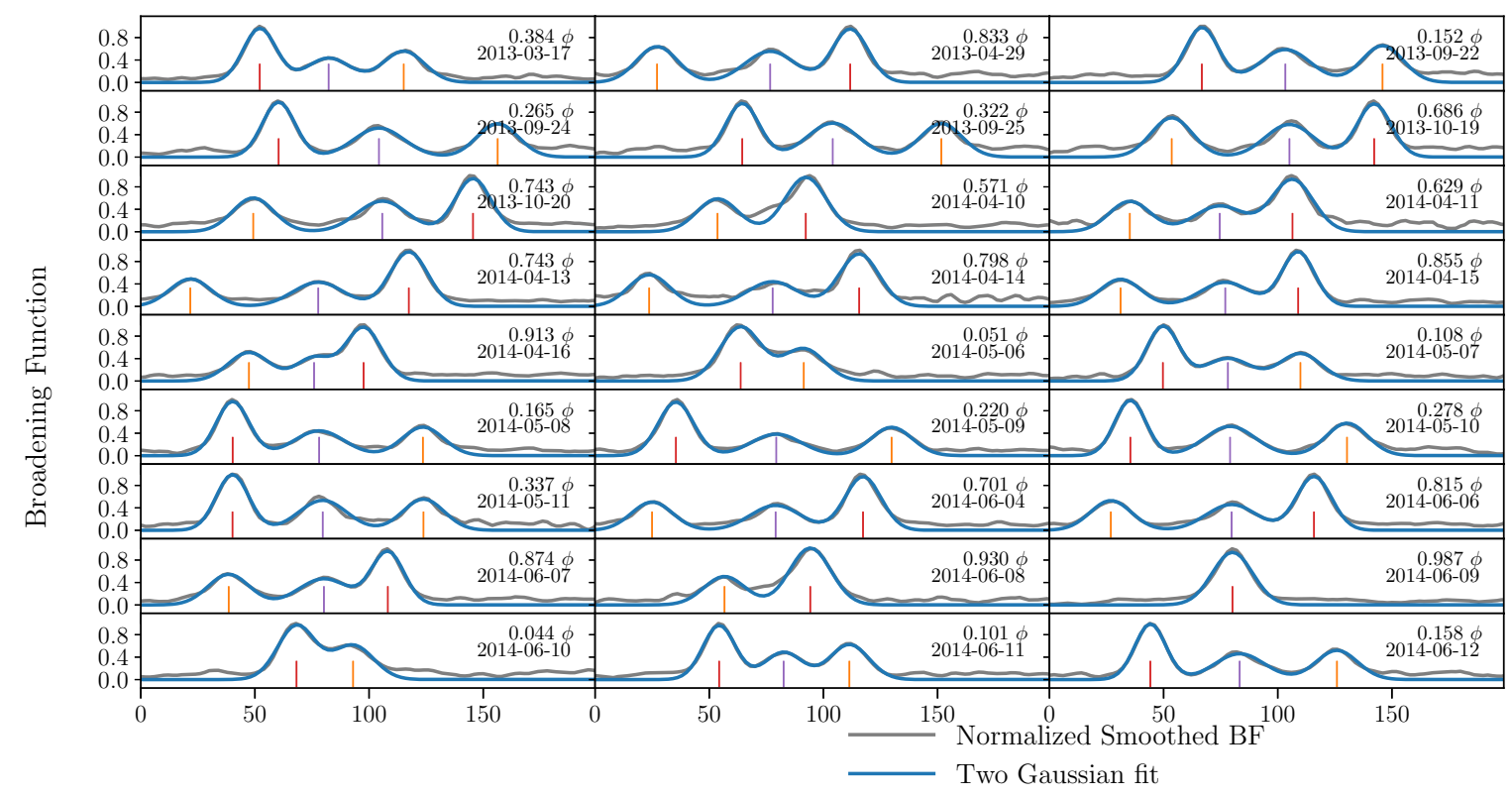

Uncorrected Radial Velocity $\left(\mathrm{km} \mathrm{s}^{-1}\right)$

Figure 22. Same as Figure 16 but for KIC 6131659. In this case the primary (red) is very distinguishable from the secondary (orange). A tertiary (purple) member is visible but it does not show variance in its radial velocity component. In some panels the tertiary is not visible because it is very near to or within the primary or secondary peak. 


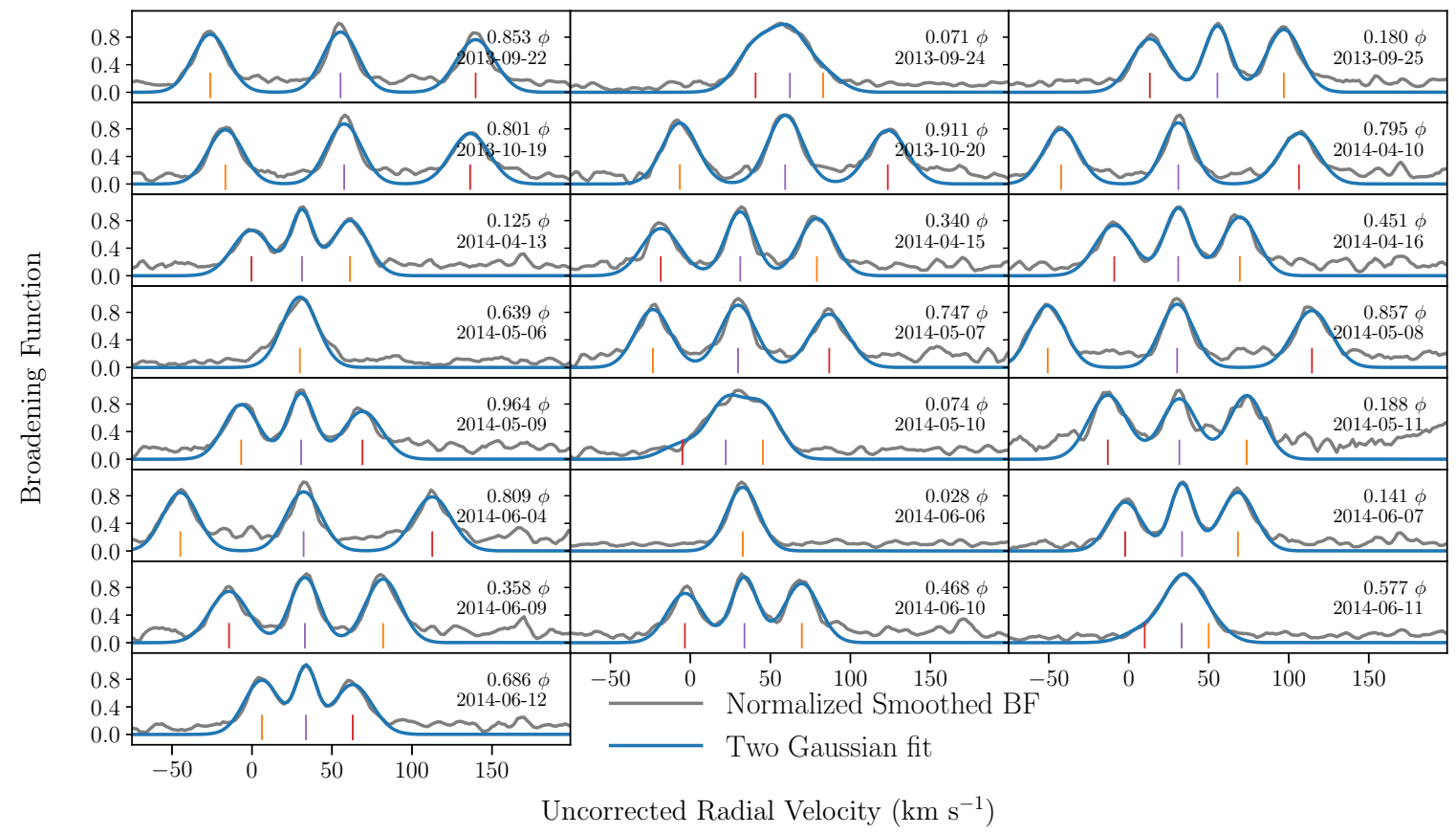

Figure 23. Same as Figure 16 but for KIC 6781535. In most panels, the primary (red) is very distinguishable from the secondary (orange). A tertiary member is present (purple) but is not RV variant. 
Table 4. Measured radial velocities from APOGEE spectra

\begin{tabular}{|c|c|c|c|}
\hline Time (BJD) & Orbital Phase & $v_{1}\left(\mathrm{~km} \mathrm{~s}^{-1}\right)$ & $v_{2}\left(\mathrm{~km} \mathrm{~s}^{-1}\right)$ \\
\hline \multicolumn{4}{|c|}{ KIC 5285607} \\
\hline 2455813.69967 & 0.040 & $41.404 \pm 0.101$ & $86.618 \pm 0.173$ \\
\hline 2455823.72647 & 0.611 & $118.406 \pm 0.061$ & $-3.961 \pm 0.108$ \\
\hline 2455840.66112 & 0.954 & $88.218 \pm 0.067$ & $31.481 \pm 0.109$ \\
\hline 2455849.57835 & 0.241 & $-24.927 \pm 0.060$ & $162.515 \pm 0.105$ \\
\hline 2455851.64874 & 0.772 & $148.087 \pm 0.060$ & $\cdots$ \\
\hline 2455866.56945 & 0.598 & $113.109 \pm 0.063$ & $2.025 \pm 0.114$ \\
\hline \multicolumn{4}{|c|}{ KIC 6864859} \\
\hline 2456557.73263 & 0.194 & $107.492 \pm 0.058$ & $\ldots$ \\
\hline 2456559.72256 & 0.243 & $110.939 \pm 0.079$ & $78.670 \pm 0.069$ \\
\hline 2456560.72029 & 0.267 & $112.129 \pm 0.062$ & $77.683 \pm 0.056$ \\
\hline 2456584.63147 & 0.852 & $74.820 \pm 0.060$ & $117.933 \pm 0.063$ \\
\hline 2456585.62998 & 0.877 & $57.225 \pm 0.064$ & $133.366 \pm 0.052$ \\
\hline 2456760.90512 & 0.164 & $106.432 \pm 0.072$ & $80.051 \pm 0.077$ \\
\hline 2456761.87222 & 0.188 & $108.904 \pm 0.073$ & $78.499 \pm 0.079$ \\
\hline 2456762.86801 & 0.213 & $109.800 \pm 0.057$ & $77.234 \pm 0.048$ \\
\hline 2456763.88053 & 0.237 & $111.281 \pm 0.053$ & $76.175 \pm 0.054$ \\
\hline 2456787.80872 & 0.823 & $81.571 \pm 0.078$ & $104.978 \pm 0.091$ \\
\hline 2456788.84246 & 0.848 & $76.011 \pm 0.053$ & $116.930 \pm 0.062$ \\
\hline 2456812.75131 & 0.433 & $114.944 \pm 0.053$ & $73.779 \pm 0.051$ \\
\hline 2456814.75480 & 0.482 & $114.257 \pm 0.049$ & $73.825 \pm 0.050$ \\
\hline 2456815.78485 & 0.507 & $113.825 \pm 0.053$ & $73.899 \pm 0.049$ \\
\hline 2456816.76560 & 0.531 & $113.034 \pm 0.061$ & $73.480 \pm 0.061$ \\
\hline 2456817.76131 & 0.555 & $112.440 \pm 0.056$ & $74.591 \pm 0.048$ \\
\hline 2456818.76390 & 0.580 & $111.523 \pm 0.056$ & $75.498 \pm 0.054$ \\
\hline 2456819.76154 & 0.604 & $110.927 \pm 0.058$ & $75.851 \pm 0.053$ \\
\hline 2456820.75533 & 0.629 & $109.049 \pm 0.059$ & $75.654 \pm 0.066$ \\
\hline \multicolumn{4}{|c|}{ KIC 6778289} \\
\hline 2456557.73261 & 0.635 & $47.229 \pm 0.045$ & $107.444 \pm 0.045$ \\
\hline 2456559.72254 & 0.701 & $38.941 \pm 0.049$ & $117.686 \pm 0.054$ \\
\hline 2456560.72027 & 0.734 & $36.535 \pm 0.046$ & $122.226 \pm 0.053$ \\
\hline 2456584.63145 & 0.528 & $60.403 \pm 0.059$ & $87.443 \pm 0.058$ \\
\hline 2456585.62996 & 0.561 & $57.486 \pm 0.047$ & $94.149 \pm 0.053$ \\
\hline 2456757.89237 & 0.278 & $101.533 \pm 0.048$ & $31.407 \pm 0.053$ \\
\hline 2456760.90514 & 0.378 & $85.993 \pm 0.051$ & $54.705 \pm 0.056$ \\
\hline 2456762.86803 & 0.443 & $72.743 \pm 0.049$ & $\ldots$ \\
\hline 2456763.88055 & 0.477 & $72.718 \pm 0.052$ & $\cdots$ \\
\hline 2456783.83502 & 0.139 & $115.038 \pm 0.049$ & $12.937 \pm 0.055$ \\
\hline 2456784.82136 & 0.172 & $113.771 \pm 0.046$ & $16.022 \pm 0.055$ \\
\hline 2456785.82484 & 0.205 & $111.263 \pm 0.044$ & $18.581 \pm 0.051$ \\
\hline 2456786.79785 & 0.237 & $108.112 \pm 0.045$ & $22.629 \pm 0.048$ \\
\hline 2456787.80874 & 0.271 & $102.199 \pm 0.047$ & $29.240 \pm 0.050$ \\
\hline 2456788.84248 & 0.305 & $98.364 \pm 0.046$ & $36.995 \pm 0.044$ \\
\hline 2456814.75483 & 0.165 & $113.882 \pm 0.048$ & $15.109 \pm 0.053$ \\
\hline 2456815.78487 & 0.200 & $111.327 \pm 0.047$ & $17.508 \pm 0.052$ \\
\hline 2456816.76563 & 0.232 & $107.622 \pm 0.050$ & $21.517 \pm 0.052$ \\
\hline 2456818.76392 & 0.298 & $97.639 \pm 0.048$ & $35.617 \pm 0.053$ \\
\hline
\end{tabular}

Table 4 continued 
Table 4 (continued)

\begin{tabular}{|c|c|c|c|}
\hline Time (BJD) & Orbital Phase & $v_{1}\left(\mathrm{~km} \mathrm{~s}^{-1}\right)$ & $v_{2}\left(\mathrm{~km} \mathrm{~s}^{-1}\right)$ \\
\hline 2456819.76156 & 0.331 & $93.260 \pm 0.046$ & $42.669 \pm 0.050$ \\
\hline 2456820.75535 & 0.364 & $87.120 \pm 0.048$ & $50.657 \pm 0.054$ \\
\hline \multicolumn{4}{|c|}{ KIC 6449358} \\
\hline 2456557.73275 & 0.431 & $47.972 \pm 0.092$ & $\cdots$ \\
\hline 2456559.72268 & 0.775 & $116.332 \pm 0.066$ & $\cdots$ \\
\hline 2456584.63158 & 0.087 & $44.828 \pm 0.067$ & $\cdots$ \\
\hline 2456585.63008 & 0.260 & $20.640 \pm 0.056$ & $\cdots$ \\
\hline 2456757.89224 & 0.080 & $46.407 \pm 0.069$ & $\cdots$ \\
\hline 2456760.90501 & 0.601 & $96.766 \pm 0.090$ & $\cdots$ \\
\hline 2456761.87212 & 0.769 & $116.275 \pm 0.066$ & $\cdots$ \\
\hline 2456763.88043 & 0.116 & $36.021 \pm 0.056$ & $\cdots$ \\
\hline 2456784.82126 & 0.741 & $115.987 \pm 0.057$ & $\cdots$ \\
\hline 2456787.80865 & 0.258 & $20.638 \pm 0.057$ & $\cdots$ \\
\hline 2456815.78483 & 0.101 & $40.477 \pm 0.069$ & $\cdots$ \\
\hline 2456816.76558 & 0.271 & $21.538 \pm 0.066$ & $\cdots$ \\
\hline 2456818.76389 & 0.617 & $101.418 \pm 0.065$ & $\cdots$ \\
\hline 2456819.76152 & 0.790 & $114.484 \pm 0.060$ & $\cdots$ \\
\hline \multicolumn{4}{|c|}{ KIC 4285087} \\
\hline 2455813.69984 & 0.864 & $154.531 \pm 0.062$ & $25.294 \pm 0.060$ \\
\hline 2455823.72663 & 0.099 & $43.398 \pm 0.060$ & $140.891 \pm 0.056$ \\
\hline 2455840.66127 & 0.874 & $150.887 \pm 0.057$ & $31.327 \pm 0.058$ \\
\hline 2455849.57849 & 0.862 & $154.496 \pm 0.056$ & $25.174 \pm 0.057$ \\
\hline 2455851.64888 & 0.323 & $17.397 \pm 0.057$ & $167.332 \pm 0.062$ \\
\hline 2455866.56955 & 0.649 & $158.478 \pm 0.060$ & $22.212 \pm 0.057$ \\
\hline \multicolumn{4}{|c|}{ KIC 6131659} \\
\hline 2456368.99876 & 0.384 & $62.544 \pm 0.048$ & $125.604 \pm 0.091$ \\
\hline 2456411.91961 & 0.833 & $125.989 \pm 0.045$ & $41.382 \pm 0.070$ \\
\hline 2456557.73279 & 0.152 & $55.739 \pm 0.042$ & $134.879 \pm 0.069$ \\
\hline 2456559.72271 & 0.265 & $49.076 \pm 0.041$ & $145.012 \pm 0.076$ \\
\hline 2456560.72045 & 0.322 & $52.988 \pm 0.043$ & $140.191 \pm 0.075$ \\
\hline 2456584.63160 & 0.686 & $128.199 \pm 0.045$ & $39.531 \pm 0.064$ \\
\hline 2456585.63010 & 0.743 & $131.456 \pm 0.045$ & $35.183 \pm 0.075$ \\
\hline 2456757.89221 & 0.571 & $105.771 \pm 0.047$ & $67.116 \pm 0.077$ \\
\hline 2456758.90157 & 0.629 & $120.004 \pm 0.052$ & $48.763 \pm 0.084$ \\
\hline 2456760.90499 & 0.743 & $131.105 \pm 0.044$ & $35.406 \pm 0.092$ \\
\hline 2456761.87209 & 0.798 & $129.464 \pm 0.048$ & $37.476 \pm 0.080$ \\
\hline 2456762.86788 & 0.855 & $122.805 \pm 0.042$ & $45.003 \pm 0.093$ \\
\hline 2456763.88040 & 0.913 & $111.491 \pm 0.099$ & $61.304 \pm 0.105$ \\
\hline 2456783.83490 & 0.051 & $78.027 \pm 0.051$ & $105.677 \pm 0.086$ \\
\hline 2456784.82125 & 0.108 & $63.945 \pm 0.053$ & $124.186 \pm 0.111$ \\
\hline 2456785.82473 & 0.165 & $54.481 \pm 0.042$ & $137.809 \pm 0.089$ \\
\hline 2456786.79774 & 0.220 & $49.620 \pm 0.044$ & $144.068 \pm 0.089$ \\
\hline 2456787.80864 & 0.278 & $49.600 \pm 0.040$ & $144.493 \pm 0.078$ \\
\hline 2456788.84238 & 0.337 & $54.269 \pm 0.041$ & $137.917 \pm 0.082$ \\
\hline 2456812.75129 & 0.701 & $129.389 \pm 0.044$ & $36.993 \pm 0.089$ \\
\hline 2456814.75479 & 0.815 & $127.626 \pm 0.044$ & $38.646 \pm 0.085$ \\
\hline 2456815.78484 & 0.874 & $119.797 \pm 0.047$ & $50.101 \pm 0.083$ \\
\hline 2456816.76560 & 0.930 & $105.694 \pm 0.045$ & $68.143 \pm 0.089$ \\
\hline 2456817.76131 & 0.987 & $91.467 \pm 0.048$ & $\cdots$ \\
\hline
\end{tabular}

Table 4 continued 
Table 4 (continued)

\begin{tabular}{|c|c|c|c|}
\hline Time (BJD) & Orbital Phase & $v_{1}\left(\mathrm{~km} \mathrm{~s}^{-1}\right)$ & $v_{2}\left(\mathrm{~km} \mathrm{~s}^{-1}\right)$ \\
\hline 2456818.76390 & 0.044 & $79.392 \pm 0.046$ & $\ldots$ \\
\hline 2456819.76154 & 0.101 & $65.338 \pm 0.047$ & $122.387 \pm 0.086$ \\
\hline 2456820.75533 & 0.158 & $55.054 \pm 0.040$ & $136.794 \pm 0.088$ \\
\hline \multicolumn{4}{|c|}{ KIC 6781535} \\
\hline 2456557.73097 & 0.853 & $128.940 \pm 0.057$ & $-37.069 \pm 0.049$ \\
\hline 2456559.72097 & 0.071 & $29.463 \pm 0.109$ & $69.329 \pm 0.204$ \\
\hline 2456560.71874 & 0.180 & $1.983 \pm 0.057$ & $85.464 \pm 0.045$ \\
\hline 2456584.63091 & 0.801 & $122.687 \pm 0.060$ & $-30.426 \pm 0.052$ \\
\hline 2456585.62946 & 0.911 & $109.784 \pm 0.057$ & $-20.436 \pm 0.047$ \\
\hline 2456757.89316 & 0.795 & $119.307 \pm 0.060$ & $-29.253 \pm 0.052$ \\
\hline 2456760.90580 & 0.125 & $12.366 \pm 0.076$ & $75.156 \pm 0.060$ \\
\hline 2456762.86860 & 0.340 & $-4.835 \pm 0.064$ & $92.365 \pm 0.049$ \\
\hline 2456763.88108 & 0.451 & $4.541 \pm 0.060$ & $82.610 \pm 0.049$ \\
\hline 2456783.83465 & 0.639 & $\cdots$ & $43.811 \pm 0.041$ \\
\hline 2456784.82095 & 0.747 & $100.401 \pm 0.057$ & $-9.574 \pm 0.049$ \\
\hline 2456785.82438 & 0.857 & $128.143 \pm 0.053$ & $-37.060 \pm 0.046$ \\
\hline 2456786.79735 & 0.964 & $82.332 \pm 0.064$ & $7.094 \pm 0.053$ \\
\hline 2456787.80820 & 0.074 & $23.165 \pm 0.158$ & $62.731 \pm 0.119$ \\
\hline 2456788.84189 & 0.188 & $1.170 \pm 0.047$ & $86.509 \pm 0.045$ \\
\hline 2456812.74399 & 0.809 & $124.321 \pm 0.056$ & $-33.597 \pm 0.049$ \\
\hline 2456814.75319 & 0.028 & $\cdots$ & $43.896 \pm 0.046$ \\
\hline 2456815.78320 & 0.141 & $8.643 \pm 0.068$ & $79.192 \pm 0.050$ \\
\hline 2456817.75959 & 0.358 & $-3.732 \pm 0.059$ & $92.410 \pm 0.045$ \\
\hline 2456818.76215 & 0.468 & $7.142 \pm 0.064$ & $80.126 \pm 0.049$ \\
\hline 2456819.75975 & 0.577 & $20.702 \pm 0.354$ & $57.626 \pm 0.204$ \\
\hline 2456820.75351 & 0.686 & $73.353 \pm 0.070$ & $15.883 \pm 0.061$ \\
\hline
\end{tabular}

\section{REFERENCES}

Astropy Collaboration, Robitaille, T. P., Tollerud, E. J., et al. 2013, A\&A, 558, A33

Bailer-Jones, C. A. L., Rybizki, J., Fouesneau, M., Mantelet, G., \& Andrae, R. 2018, The Astronomical Journal, 156, 58

Bayless, A. J., \& Orosz, J. A. 2006, The Astrophysical Journal, 651, 1155

Borkovits, T., Hajdu, T., Sztakovics, J., et al. 2016, MNRAS, 455, 4136

Borucki, W. J., Koch, D., Basri, G., et al. 2010, Science, 327,977

Bovy, J. 2016, The Astrophysical Journal, 817, 49

Cohen, M., Wheaton, W. A., \& Megeath, S. T. 2003, AJ, 126,1090

Conroy, K. E., Prša, A., Stassun, K. G., et al. 2014, AJ, 147,45

Czesla, S. 2018, ,

Dotter, A., Chaboyer, B., Jevremović, D., et al. 2008, The Astrophysical Journal Supplement Series, 178, 89
El-Badry, K., Rix, H.-W., Ting, Y.-S., et al. 2017, MNRAS, 473,5043

Fleming, S. W., Mahadevan, S., Deshpande, R., et al. 2015, The Astronomical Journal, 149, 143

Foreman-Mackey, D., Hogg, D. W., Lang, D., \& Goodman, J. 2013, PASP, 125, 306

Foreman-Mackey, D., Hogg, D. W., Lang, D., \& Goodman, J. 2013, PASP, 125, 306

Gaulme, P., McKeever, J., Jackiewicz, J., et al. 2016, The Astrophysical Journal, 832, 121

Ginsburg, A. 2017, ,

Hunter, J. D. 2007, Computing In Science \& Engineering, 9,90

Husser, T.-O., von Berg, S. W., Dreizler, S., et al. 2013, Astronomy \& Astrophysics, 553, A6

Jones, E., Oliphant, T., Peterson, P., et al. 2001, SciPy: Open source scientific tools for Python, ,

Kipping, D. M. 2010, Monthly Notices of the Royal Astronomical Society, 408, 1758

Kipping, D. M. 2013, Astronomy and Astrophysics, 154, 216 
Kirk, B., Conroy, K., Prsa, A., et al. 2016, The

Astronomical Journal, 151, 68

Kozai, Y. 1962, The Astronomical Journal, 67, 591

Lehmann, H., Zechmeister, M., Dreizler, S., Schuh, S., \& Kanzler, R. 2012, Astronomy and Astrophysics, 541, A105

Lu, W., Rucinski, S. M., \& OgŁłoza, W. 2001, The Astronomical Journal, 122, 402

Majewski, S. R., Schiavon, R. P., Frinchaboy, P. M., et al. 2015, arXiv.org, 1509.05420v1

Mandel, K., \& Agol, E. 2002, The Astrophysical Journal, 580, L171

Matson, R. A., Gies, D. R., Guo, Z., \& Williams, S. J. 2017, The Astronomical Journal, 154, 216

McKinney, W. 2010, in Proceedings of the 9th Python in Science Conference, ed. S. van der Walt \& J. Millman, 51 $-56$

Morton, T. D., Bryson, S. T., Coughlin, J. L., et al. 2016, The Astrophysical Journal, 822, 86

Newville, M., Stensitzki, T., Allen, D. B., et al. 2016, Astrophysics Source Code Library, ascl:1606.014

Nidever, D. L., Holtzman, J. A., Prieto, C. A., et al. 2015, The Astronomical Journal, 150, 173

Pérez, A. E. G., Prieto, C. A., Holtzman, J. A., et al. 2016, The Astronomical Journal, 151, 144

Pinsonneault, M. H., Elsworth, Y., Epstein, C., et al. 2014, The Astrophysical Journal Supplement Series, 215, 19

Pinsonneault, M. H., Elsworth, Y. P., Tayar, J., et al. 2018, ArXiv e-prints, arXiv:1804.09983

Price-Whelan, A., Mechev, A., \& jumeroag. 2018, adrn/makecite: v0.1, , , doi:10.5281/zenodo.1343295
Price-Whelan, A. M., Sipőcz, B. M., Günther, H. M., et al. 2018, AJ, 156, 123

Rappaport, S., Deck, K., Levine, A., et al. 2013, ApJ, 768, 33

Rawls, M. L., Gaulme, P., McKeever, J., et al. 2016, The Astrophysical Journal, 818, 108

Rucinski, S. M. 1992, The Astronomical Journal, 104, 1968

- 1999, International Astronomical Union Colloquium, 170,82

—. 2002, The Astronomical Journal, 124, 1746

—. 2004, Symposium - International Astronomical Union, 215, 17

Skrutskie, M. F., Cutri, R. M., Stiening, R., et al. 2006, AJ, 131,1163

Stassun, K. G., Collins, K. A., \& Gaudi, B. S. 2017, AJ, 153,136

Stassun, K. G., Mathieu, R. D., \& Valenti, J. A. 2007, The Astrophysical Journal, 664, 1154

Stassun, K. G., \& Torres, G. 2018, ApJ, 862, 61

Thompson, S. E., Everett, M., Mullally, F., et al. 2012, ApJ, 753, 86

Tokovinin, A., Thomas, S., Sterzik, M., \& Udry, S. 2006, Astronomy and Astrophysics, 450, 681

Tokovinin, A. A. 1997, Astronomy Letters, 23, 727

Torres, G., Andersen, J., \& Giménez, A. 2010, A\&A Rv, 18,67

Torres, G., Curtis, J. L., Vanderburg, A., Kraus, A. L., \& Rizzuto, A. 2018, arXiv.org, arXiv:1808.07482

Van Der Walt, S., Colbert, S. C., \& Varoquaux, G. 2011, ArXiv e-prints, arXiv:1102.1523

Windemuth, D., Agol, E., Ali, A., \& Kiefer, F. 2018, MNRAS 